\title{
CONVERGENCE OF MASS REDISTRIBUTION METHOD FOR THE ONE-DIMENSIONAL WAVE EQUATION WITH A UNILATERAL CONSTRAINT AT THE BOUNDARY
}

\author{
Farshid Dabaghi ${ }^{1}$, Adrien Petrov ${ }^{1}$, Jérôme Pousin ${ }^{1}$ And Yves Renard ${ }^{1}$
}

\begin{abstract}
This paper focuses on a one-dimensional wave equation being subjected to a unilateral boundary condition. Under appropriate regularity assumptions on the initial data, a new proof of existence and uniqueness results is proposed. The mass redistribution method, which is based on a redistribution of the body mass such that there is no inertia at the contact node, is introduced and its convergence is proved. Finally, some numerical experiments are reported.
\end{abstract}

Mathematics Subject Classification. 35L85, 35L05, 65N30, 74M15.

Received January 23, 2013. Revised June 11, 2013.

Published online July 8, 2014.

\section{INTRODUCTION}

We consider an elastic bar of length $L$ vibrating vertically. More precisely, one end of the bar is free to move, as long as it does not hit a material obstacle, while the other end is clamped (see Fig. 1). The obstacle constrains the displacement of the extremity to be greater than or equal to 0 .

We describe now the mathematical situation. We assume that the material of the bar is homogeneous and satisfies the theory of small deformations. Let $x$ be the spatial coordinate along the bar, with the origin at the material obstacle, let $u(x, t)$ be the displacement at time $t \in[0, T], T>0$, of the material point of spatial coordinate $x \in[0, L]$. Then the mathematical problem can be formulated as follows:

$$
\ddot{u}(x, t)-u^{\prime \prime}(x, t)=0, \quad(x, t) \in(0, L) \times(0, T),
$$

with Cauchy initial data

$$
u(x, 0)=u^{0}(x) \quad \text { and } \quad \dot{u}(x, 0)=v^{0}(x), \quad x \in(0, L),
$$

and Signorini and Dirichlet boundary conditions at $x=0$ and $x=L$, respectively,

$$
0 \leq u(0, t) \perp u^{\prime}(0, t) \leq 0 \quad \text { and } \quad u(L, t)=0, \quad t \in[0, T],
$$

\footnotetext{
Keywords and phrases. Existence, uniqueness, convergence, mass redistribution method, variational inequality, unilateral contact.

1 Université de Lyon, CNRS, INSA-Lyon, Institut Camille Jordan UMR 5208, 20 Avenue A. Einstein, 69621 Villeurbanne, France. farshid.dabaghi@insa-lyon.fr; apetrov@math.univ-lyon1.fr

jerome.pousin@insa-lyon.fr; Yves.Renard@insa-lyon.fr
} 


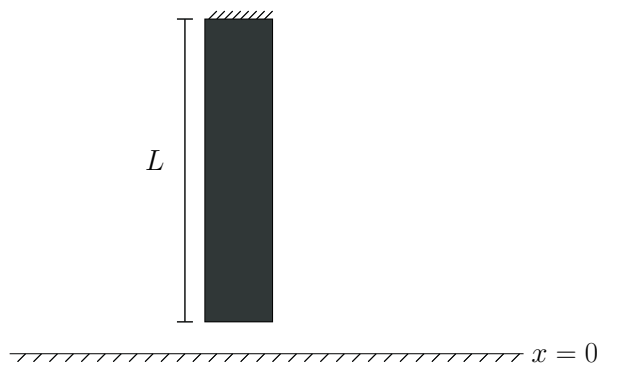

FiguRE 1. An elastic bar vibrating on impacting obstacle.

where $\dot{u} \stackrel{\text { def }}{=} \frac{\partial u}{\partial t}$ and $u^{\prime} \stackrel{\text { def }}{=} \frac{\partial u}{\partial x}$. The orthogonality has the natural meaning; namely if we have enough regularity, it means that the product $u(0, \cdot) u^{\prime}(0, \cdot)$ vanishes almost everywhere at the boundary. If it is not the case, the above inequality is integrated on an appropriate set of test functions, leading to a weak formulation for the unilateral condition.

Observe that from mathematical viewpoint, the Signorini conditions mean that when the bar touches the obstacle in $x=0$, its reaction can be only upwards, so that $u^{\prime}(0, t) \leq 0$ on the set $\{t: u(0, t)=0\}$. While in the case where the bar does not touch the obstacle, its end is free to move. More precisely, we have $u^{\prime}(0, t)=0$ on the set $\{t: u(0, t)>0\}$.

We suppose that the initial displacement $u^{0}$ belongs to the Sobolev space $\mathrm{H}^{1}(0, L)$ and satisfies the compatibility conditions, i.e. $u^{0}(L)=0$ and $u^{0}(0) \geq 0$ and the initial velocity $v^{0}$ belongs to $\mathrm{L}^{2}(0, L)$.

We describe now the weak formulation of the problem. To this aim, we denote by $\mathbb{K}$ the following convex set:

$$
\mathbb{K} \stackrel{\text { def }}{=}\left\{u \in \mathbb{H}_{2}: u(\cdot, t) \in K \text { for almost every } t\right\},
$$

where $\mathbb{H}_{2} \stackrel{\text { def }}{=}\left\{u \in \mathrm{L}^{2}(0, T ; V): \dot{u} \in \mathrm{L}^{2}\left(0, T ; \mathrm{L}^{2}(0, L)\right)\right\}$ and $K \stackrel{\text { def }}{=}\{u \in V: u(0) \geq 0\}$ with $V \stackrel{\text { def }}{=}\left\{u \in \mathrm{H}^{1}(0, L)\right.$ : $u(L)=0\}$.

Then the weak formulation associated to (1.1)-(1.3) is obtained by multiplying (1.1) by $v-u, v \in \mathbb{K}$ and by integrating formally this result over $Q_{T} \stackrel{\text { def }}{=}(0, L) \times(0, T)$ :

$$
\left\{\begin{array}{l}
\text { find } u \in \mathbb{K} \text { such that } \\
-\int_{0}^{L} v^{0}\left(v(\cdot, 0)-u^{0}\right) \mathrm{d} x-\int_{Q_{T}} \dot{u}(\dot{v}-\dot{u}) \mathrm{d} x \mathrm{~d} t+\int_{Q_{T}} u^{\prime}\left(v^{\prime}-u^{\prime}\right) \mathrm{d} x \mathrm{~d} t \geq 0 \\
\text { for all } v \in \mathbb{K} \text { for which there exists } \zeta>0 \text { with } v=u \text { for } t \geq T-\zeta .
\end{array}\right.
$$

The weak formulation (1.4) is derived from [32] where the contact conditions are given in a slightly different context. Existence and uniqueness results are obtained for a vibrating string with concave obstacle in onedimensional space in [31] and for a wave equation with unilateral constraint at the boundary in a half space of $\mathbb{R}^{N}$ in [22]. An existence result for a wave equation in a $\mathrm{C}^{2}$ regular bounded domain constrained by an obstacle at the boundary in $\mathbb{R}^{N}$ for $N \geq 2$ is proven in [19].

The paper is organized as follows. In Section 2, the problem (1.1)-(1.3) is reformulated as a differential inclusion problem by using characteristic method, which is a crucial ingredient to prove the uniqueness result. Then, the rest of this section is devoted to the proof of existence and uniqueness results as well as to the energy balance. In Section 3, the equivalence between the weak formulation associated to (1.1)-(1.3) and the differential inclusion obtained in Section 2 is established. Then, in Section 4, a mass redistribution method is introduced and its convergence is proved. This method is based on a redistribution of the body mass such that there is no inertia at the contact node (see $[13,16]$ ). Finally, some numerical examples are reported and analyzed in Section 5. More precisely, the energy with and without the mass redistribution method are compared as well as the approximated solution associated to the mass redistribution method and an exact solution. 


\section{Existence AND Uniqueness RESUlts BY USING THE CHARACTERISTIC METHOD}

This section is devoted to the proof of existence and uniqueness results for the problem (1.1)-(1.3). The first step consists in rewriting (1.1)-(1.3) as a differential inclusion problem by using the characteristic method. To this aim, we introduce the following notations:

$$
\xi \stackrel{\text { def }}{=} x+t \quad \text { and } \quad \eta \stackrel{\text { def }}{=} x-t .
$$

Therefore the chain rule gives

$$
\frac{\partial^{2} u}{\partial x^{2}}=\frac{\partial^{2} u}{\partial \xi^{2}}+2 \frac{\partial^{2} u}{\partial \xi \partial \eta}+\frac{\partial^{2} u}{\partial \eta^{2}} \quad \text { and } \quad \frac{\partial^{2} u}{\partial t^{2}}=\frac{\partial^{2} u}{\partial \xi^{2}}-2 \frac{\partial^{2} u}{\partial \xi \partial \eta}+\frac{\partial^{2} u}{\partial \eta^{2}},
$$

which by using (1.1) implies that $\frac{\partial^{2} u}{\partial \xi \partial \eta}$ vanishes. Thus we may conclude that

$$
u(\xi, \eta)=p(\xi)+q(\eta)
$$

where $p$ and $q$ are two differentiable functions such that

$$
u(x, t)=p(x+t)+q(x-t) .
$$

In particular, taking $t=0$ and using the initial data $u^{0}$ and $v^{0}$, we get

$$
p(x)+q(x)=u^{0}(x) \text { and } \quad p^{\prime}(x)-q^{\prime}(x)=v^{0}(x),
$$

which by integration gives

$$
p(\xi)=\frac{u^{0}(\xi)}{2}+\frac{1}{2} \int_{0}^{\xi} v^{0}(\bar{x}) \mathrm{d} \bar{x} \quad \text { and } \quad q(\eta)=\frac{u^{0}(\eta)}{2}-\frac{1}{2} \int_{0}^{\eta} v^{0}(\bar{x}) \mathrm{d} \bar{x}
$$

for all $\xi$ and $\eta$ belonging to $[0, L]$. According to (2.1), the boundary conditions (1.3) can be rewritten as follows:

$$
0 \leq p(t)+q(-t) \perp p^{\prime}(t)+q^{\prime}(-t) \leq 0 \text { and } \quad p(L+t)+q(L-t)=0
$$

for all $t$ belonging to $[0, T]$. Thanks to the above identity, we may extend $p(t)$ for all $t \in[L, 2 L]$, i.e. we have

$$
p(L+t)=-q(L-t)
$$

for all $t$ belonging to $[0, L]$. If we choose $t^{\prime}=L+t$, we get $p\left(t^{\prime}\right)=-q\left(2 L-t^{\prime}\right)$. We already have the solution for $q(t)$ with $0 \leq t \leq L$ and if $L \leq t^{\prime} \leq 2 L$, we can obtain $p\left(t^{\prime}\right)$ by observing that $0 \leq 2 L-t^{\prime} \leq L$ and by using $q(2 L-t)$, it comes that

$$
p(t)=-\frac{u^{0}(2 L-t)}{2}+\frac{1}{2} \int_{0}^{2 L-t} v^{0}(\bar{x}) \mathrm{d} \bar{x}
$$

for all $t$ belonging to $[L, 2 L]$.

Let us introduce the multivalued function $J_{N}: \mathbb{R} \rightarrow \mathcal{P}(\overline{\mathbb{R}}) \backslash \emptyset$ defined by

$$
J_{N}(x) \stackrel{\text { def }}{=}\left\{\begin{array}{lll}
\{0\} & \text { if } & x<0 \\
{[0,+\infty)} & \text { if } & x=0 \\
\emptyset & \text { if } \quad x>0
\end{array}\right.
$$

where $\mathcal{P}(\overline{\mathbb{R}})$ is the set of all subsets of $\overline{\mathbb{R}}$. More precisely, $J_{N}(x)$ is the subdifferential of the indicator function $\partial I_{(-\infty, 0]}(x)$ defined by

$$
I_{(-\infty, 0]}(x) \stackrel{\text { def }}{=}\left\{\begin{array}{lll}
0 & \text { if } & x \in(-\infty, 0], \\
+\infty & \text { if } & x \notin(-\infty, 0] .
\end{array}\right.
$$


Obviously, $I_{(-\infty, 0]}$ is a lower semi-continuous and convex function, for further details the reader is referred to [6]. Then, the inequalities in (2.4) can be rewritten as follows

$$
\left.p^{\prime}(t)+q^{\prime}(-t) \in-J_{N}(-p(t)-q(-t))\right) .
$$

Note that at this stage, $q(\eta), \eta \in[-L, 0]$, is the unique unknown of (2.6). We define now

$$
f(t) \stackrel{\text { def }}{=}-p(t)-q(-t) \text {. }
$$

We insert (2.7) into (2.6) to get

$$
f^{\prime}(t) \in-J_{N}(f(t))-2 p^{\prime}(t) .
$$

Finally, we find the following Cauchy problem

$$
\begin{aligned}
& f^{\prime}(t) \in-J_{N}(f(t))-2 p^{\prime}(t) \quad \text { a.e. } \quad t \in(0, L), \\
& f(0)=-u^{0}(0) .
\end{aligned}
$$

Observe that the Cauchy problem (2.8) formally is equivalent to (1.1)-(1.3). Note that the existence and uniqueness results in half-space, with some appropriate conditions on $u^{0}$ and $v^{0}$, were established in [22]. The proof of Theorem 2.1 is rather classical. However for the reader convenience, this proof is given in the Appendix A.

Theorem 2.1 (Existence and uniqueness results). Assume that $p$ is bounded in $\mathrm{W}^{1,1}(0, L)$. Then the Cauchy problem (2.8) admits a unique absolutely continuous solution.

We introduce now some new notations: let $\stackrel{\circ}{X}$ and $\partial X$ be the interior and boundary of the set $X$, respectively, and let

$$
I \stackrel{\text { def }}{=}\{t \in[0, L]: f(t)=0\} \quad \text { and } \quad J \stackrel{\text { def }}{=}\{t \in[0, L]: f(t)<0\} .
$$

In the sequel, the notations for the constants introduced in the proofs are only valid in the proof. The aim of the next lemma is to prove further regularity results for the solution $f$ of Problem (2.8).

Lemma 2.2 (regularity result). Assume that $p$ is bounded in $\mathrm{H}^{1}(0, L)$. Then the solution $f$ to problem (2.8) is bounded in $\mathrm{H}^{1}(0, L)$.

Proof. Note that Theorem 2.1 implies that $f$ is bounded in $\mathrm{W}^{1,1}(0, L)$. It follows that

$$
f^{\prime}(t)=0 \text { on } \stackrel{\circ}{I} \text { and } f^{\prime}(t)=-2 p^{\prime}(t) \text { on } J .
$$

Clearly, we have

$$
\int_{J}\left|f^{\prime}(t)\right|^{2} \mathrm{~d} t=\int_{J} 4\left|p^{\prime}(t)\right|^{2} \mathrm{~d} t<\infty \quad \text { and } \quad \int_{\dot{I}}\left|f^{\prime}(t)\right|^{2} \mathrm{~d} t=0 .
$$

Observe that if $t$ is an accumulation point of $\partial I$, we may deduce that there exists a sequence $t_{n}$ belonging to $I$ such that $t_{n} \rightarrow t$ so that $f\left(t_{n}\right)=0$ and $f(t)=0$. We recall the fundamental theorem of calculus for Lebesgue integral states that if $f$ is an absolutely continuous function on $[a, b], f$ is of bounded variation on $[a, b]$. Consequently, $f^{\prime}(x)$ exists for almost every $x$ belonging to $[a, b]$. For further details the reader is referred to [30], p. 160. Hence $f^{\prime}$ vanishes for almost all accumulation points of $\partial I$. It follows that $f^{\prime}(t)$ vanishes for almost every $t$ belonging to $\partial I$. Therefore, we deduce that $f^{\prime}$ is bounded in $L^{2}(0, L)$, which implies that $f$ belongs to $\mathrm{H}^{1}(0, L)$. This concludes the proof. 
It is convenient to define the following spaces:

$$
H \stackrel{\text { def }}{=} \mathrm{L}^{2}(0, L) \quad \text { and } \quad V \stackrel{\text { def }}{=}\left\{u \in \mathrm{H}^{1}(0, L): u(L)=0\right\}
$$

endowed with the norms $\|\cdot\|_{H}$ and $\|\cdot\|_{V}$. Let $(\cdot, \cdot)$ and $a(\cdot, \cdot)$ the scalar products in $H$ and $V$, respectively. This allows to define

$$
\mathbb{H}_{2} \stackrel{\text { def }}{=}\left\{u \in \mathrm{L}^{2}(0, T ; V): \dot{u} \in \mathrm{L}^{2}(0, T ; H)\right\}
$$

endowed with the norm

$$
\|u\|_{\mathbb{H}_{2}} \stackrel{\text { def }}{=}\left(\int_{0}^{T}\left(\|u(\cdot, t)\|_{V}^{2}+\|\dot{u}(\cdot, t)\|_{H}^{2}\right) \mathrm{d} t\right)^{1 / 2}
$$

and $\langle\langle\cdot, \cdot\rangle\rangle$ the duality corresponding product between $\mathbb{H}_{2}^{\prime}$ and $\mathbb{H}_{2}$. We observe that $\mathbb{H}_{2} \hookrightarrow \mathrm{C}^{0}([0, T] ; H)$ (see $[34]$ ).

Lemma 2.3. Assume that $u$ belongs to $\mathbb{H}_{2}$ and $\ddot{u}-u^{\prime \prime}$ (defined in the sense of distributions on $Q_{T}$ ) is square integrable. Then we have for all $\epsilon>0, u \in \mathrm{C}^{0}\left([0, T] ; \mathrm{H}^{1}(\epsilon, L)\right) \cap \mathrm{C}^{0}\left([0, L] ; \mathrm{H}^{1}(\epsilon, T-\epsilon)\right)$, $\dot{u} \in \mathrm{C}^{0}\left([0, T] ; \mathrm{L}^{2}(\epsilon, L)\right)$ and $u^{\prime} \in \mathrm{C}^{0}\left([0, L] ; \mathrm{L}^{2}(\epsilon, T-\epsilon)\right)$.

Proof. The proof is obtained by using the same techniques detailed in [32]. Since it is quite a routine to adapt this proof to our case, we let the verification to the reader.

The aim of the next lemma is to obtain some further regularity results for the solution $u$ to (1.4).

Lemma 2.4. Let $u$ be the solution to (1.4). Then for all $\epsilon>0, u \in \mathrm{C}^{0}\left([0, L] ; \mathrm{H}^{1}(0, T-\epsilon)\right)$ and $u^{\prime} \in$ $\mathrm{C}^{0}\left([0, L] ; \mathrm{L}^{2}(0, T-\epsilon)\right)$.

Proof. The proof of this result exploits the local energy identity inside $Q_{T}$, the reader can find a detailed proof in the Appendix as well as in [32] where a succinct proof is given.

We deal now with the energy balance. More precisely, we prove below that the energy associated to (2.8) given by

$$
\mathcal{E}(t)=\frac{1}{2} \int_{0}^{L}\left(\left|u^{\prime}(t, x)\right|^{2}+|\dot{u}(t, x)|^{2}\right) \mathrm{d} x
$$

is constant with respect to time $t$.

Lemma 2.5 (energy balance). Assume that $p$ is bounded in $\mathrm{W}^{1,1}(0, L)$. Then the solution $u$ to problem $(2.8)$ is energy conserving.

Proof. We observe first that (2.1) gives

$$
\mathcal{E}(t)=\frac{1}{2} \int_{0}^{L}\left(\left(p^{\prime}(x+t)+q^{\prime}(x-t)\right)^{2}+\left(p^{\prime}(x+t)-q^{\prime}(x-t)\right)^{2}\right) \mathrm{d} x=\int_{0}^{L}\left|p^{\prime}(x+t)\right|^{2} \mathrm{~d} x+\int_{0}^{L}\left|q^{\prime}(x-t)\right|^{2} \mathrm{~d} x .
$$

We evaluate now the two integrals on the right hand side of (2.9). We note first that (2.3), (2.5) and (2.7) lead to

$$
p^{\prime}(x+t)= \begin{cases}\frac{1}{2}\left(u^{0^{\prime}}(x+t)+v^{0}(x+t)\right), & 0 \leq x+t \leq L, \\ \frac{1}{2}\left(u^{0^{\prime}}(2 L-(x+t))-v^{0}(2 L-(x+t))\right), & L \leq x+t \leq 2 L,\end{cases}
$$

and

$$
q^{\prime}(x-t)= \begin{cases}\frac{1}{2}\left(u^{0^{\prime}}(x-t)-v^{0}(x-t)\right), & 0 \leq x-t \leq L, \\ \left(f^{\prime}(-(x-t))+p^{\prime}(-(x-t))\right), & -L \leq x-t \leq 0 .\end{cases}
$$


On the one hand, we may deduce that

$$
\begin{aligned}
\int_{0}^{L}\left|p^{\prime}(x+t)\right|^{2} \mathrm{~d} x & =\frac{1}{4} \int_{0}^{L-t}\left|u^{0^{\prime}}(x+t)+v^{0}(x+t)\right|^{2} \mathrm{~d} x+\frac{1}{4} \int_{L-t}^{L}\left|u^{0^{\prime}}(2 L-x-t)-v^{0}(2 L-x-t)\right|^{2} \mathrm{~d} x \\
& =\frac{1}{4} \int_{t}^{L}\left|u^{0^{\prime}}(x)+v^{0}(x)\right|^{2} \mathrm{~d} x+\frac{1}{4} \int_{L-t}^{L}\left|u^{0^{\prime}}(x)-v^{0}(x)\right|^{2} \mathrm{~d} x .
\end{aligned}
$$

On the other hand, by using the same kind of arguments as in the proof of Lemma 2.2, we may obtain

$$
\left|q^{\prime}(\eta)\right|^{2}=\left|f^{\prime}(-\eta)+p^{\prime}(-\eta)\right|^{2}= \begin{cases}\left|0+p^{\prime}(-\eta)\right|^{2} & \text { a.e. on } I, \\ \left|-2 p^{\prime}(-\eta)+p^{\prime}(-\eta)\right|^{2} & \text { on } J,\end{cases}
$$

for all $\eta$ belonging to $[-L, 0]$. It follows that $\left|q^{\prime}(\eta)\right|^{2}=\left|p^{\prime}(-\eta)\right|^{2}$ almost everywhere on $[-L, 0]$. Hence we have

$$
\begin{aligned}
\int_{0}^{L}\left|q^{\prime}(x-t)\right|^{2} \mathrm{~d} x & =\int_{0}^{t}\left|p^{\prime}(t-x)\right|^{2} \mathrm{~d} x+\frac{1}{4} \int_{t}^{L}\left|u^{0^{\prime}}(x-t)+v^{0}(x-t)\right|^{2} \mathrm{~d} x \\
& =\frac{1}{4} \int_{0}^{t}\left|u^{0^{\prime}}(t-x)+v^{0}(t-x)\right|^{2} \mathrm{~d} x+\frac{1}{4} \int_{t}^{L}\left|u^{0^{\prime}}(x-t)+v^{0}(x-t)\right|^{2} \mathrm{~d} x \\
& =\frac{1}{4} \int_{0}^{t}\left|u^{0^{\prime}}(x)+v^{0}(x)\right|^{2} \mathrm{~d} x+\frac{1}{4} \int_{0}^{L-t}\left|u^{0^{\prime}}(x)-v^{0}(x)\right|^{2} \mathrm{~d} x .
\end{aligned}
$$

Inserting (2.10) and (2.11) into (2.9), we get

$$
\mathcal{E}(t)=\frac{1}{4} \int_{0}^{L}\left|u^{0^{\prime}}(x)+v^{0}(x)\right|^{2} \mathrm{~d} x+\frac{1}{4} \int_{0}^{L}\left|u^{0^{\prime}}(x)-v^{0}(x)\right|^{2} \mathrm{~d} x,
$$

and the conclusion is clear.

\section{The equivalence Between the variational Formulation AND THE DIFFERENTIAL INCLUSION}

The present section is dedicated to prove the equivalence between the weak formulation (1.4) and the differential inclusion (2.8). Consequently together with the results obtained in the previous section, it is possible to deduce that (1.4) possesses a unique solution. To this aim, we introduce

$$
K \stackrel{\text { def }}{=}\{u \in V: u(0, \cdot) \geq 0\} .
$$

Lemma 3.1. Assume that $u^{0}$ and $v^{0}$ belong to $K$ and $H$, respectively. Then the unique solution to problem (2.8) define a weak solution to (1.4) for $T=L$.

Proof. The idea of the proof consists to split the domain $Q_{T}$ into four regions according to Figure 2 and to use the expression of the solution on each region to show that $u$ and $\dot{u}$ belong to $\mathrm{L}^{2}(0, T ; V)$ and to $\mathrm{L}^{2}(0, T ; H)$, respectively (see Fig. 2).

Let us go into the details. On the one hand, we observe that $x+t \in[0, L]$ and $x-t \in[-L, 0]$ in the region I while $x+t \in[L, 2 L]$ and $x-t \in[-L, 0]$ in the region II and we have

$$
\begin{aligned}
u(x, t) & =p(x+t)-p(t-x)-f(t-x), \\
u^{\prime}(x, t) & =p^{\prime}(x+t)+p^{\prime}(t-x)+f^{\prime}(t-x), \\
\dot{u}(x, t) & =p^{\prime}(x+t)-p^{\prime}(t-x)-f^{\prime}(t-x) .
\end{aligned}
$$




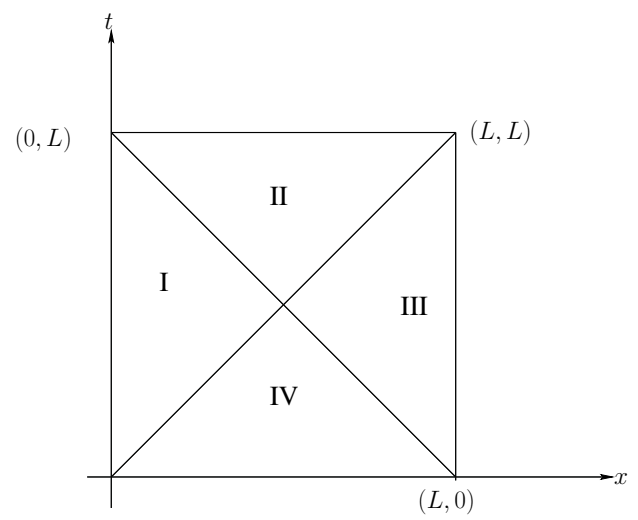

Figure 2. Four regions allowing to determine the value of $u$.

Since $u^{0} \in K, v^{0} \in H$ and (2.3)-(2.5) hold, we may infer that $p$ belongs to $\mathrm{H}^{1}(0,2 L)$ in the regions I and II. Besides, Lemma 2.2 implies that $f$ belongs to $\mathrm{H}^{1}(0, L)$. According to $(3.1)$, we conclude that

$$
\int_{0}^{L} \int_{x}^{L}\left(|u(x, t)|^{2}+\left|u^{\prime}(x, t)\right|^{2}+|\dot{u}(x, t)|^{2}\right) \mathrm{d} t \mathrm{~d} x<+\infty .
$$

On the other hand, we note that $x+t \in[L, 2 L]$ and $x-t \in[0, L]$ in the region III while $x+t \in[0, L]$ and $x-t \in[0, L]$ in the region IV and we have

$$
\begin{aligned}
u(x, t) & =p(x+t)+q(x-t), \\
u^{\prime}(x, t) & =p^{\prime}(x+t)+q^{\prime}(x-t), \\
\dot{u}(x, t) & =p^{\prime}(x+t)-q^{\prime}(x-t) .
\end{aligned}
$$

Still using the fact that $u^{0} \in K, v^{0} \in H$ and (2.3)-(2.5) hold, we may infer that $p$ and $q$ belong to $\mathrm{H}^{1}(0, L)$ in the regions III and IV. Thanks to (3.3), we may infer that

$$
\int_{0}^{L} \int_{0}^{x}\left(|u(x, t)|^{2}+\left|u^{\prime}(x, t)\right|^{2}+|\dot{u}(x, t)|^{2}\right) \mathrm{d} t \mathrm{~d} x<+\infty .
$$

Therefore, it follows from (3.2) and (3.4) that $u$ and $\dot{u}$ are bounded in $\mathrm{L}^{2}(0, T ; V)$ and $\mathrm{L}^{2}(0, T ; H)$, respectively. We deduce from Lemma 2.3 that $u(0, \cdot)$ and $u^{\prime}(0, \cdot)$ belong to $\mathrm{L}^{2}(0, T)$ and to $\mathrm{L}^{2}(\epsilon, T-\epsilon)$ for all $\epsilon>0$, respectively, and $u(\cdot, 0)$ and $\dot{u}(\cdot, 0)$ belong to $\mathrm{L}^{2}(0, L)$ and to $\mathrm{L}^{2}(\epsilon, L)$ for all $\epsilon>0$, respectively. It remains to verify that (1.4) holds. To this aim, we observe by using the notations introduced above that

$$
\begin{aligned}
& -\int_{0}^{L} v^{0}(v(x, 0)-u(x, 0)) \mathrm{d} x-\int_{Q_{T}} \dot{u}(x, t)(\dot{v}-\dot{u})(x, t) \mathrm{d} x \mathrm{~d} t+\int_{Q_{T}} u^{\prime}(x, t)\left(v^{\prime}-u^{\prime}\right)(x, t) \mathrm{d} x \mathrm{~d} t \\
= & -\int_{0}^{L} v^{0}(v(x, 0)-u(x, 0)) \mathrm{d} x-\int_{Q_{T}}\left(p^{\prime}(x+t)-q^{\prime}(x-t)\right)\left(\dot{v}(x, t)-\left(p^{\prime}(x+t)-q^{\prime}(x-t)\right)\right) \mathrm{d} x \mathrm{~d} t \\
& +\int_{Q_{T}}\left(p^{\prime}(x+t)+q^{\prime}(x-t)\right)\left(v^{\prime}(x, t)-\left(p^{\prime}(x+t)+q^{\prime}(x-t)\right)\right) \mathrm{d} x \mathrm{~d} t
\end{aligned}
$$

for all $v$ belonging to $\mathbb{K}$ such that there exists $\zeta>0$ with $v=u$ for $t \geq L-\zeta$. We evaluate each integral on the right hand side of (3.5). Thanks to (2.2), we have

$$
\int_{0}^{L} v^{0}(v(x, 0)-u(x, 0)) \mathrm{d} x=\int_{0}^{L}\left(p^{\prime}(x)-q^{\prime}(x)\right)(v(x, 0)-(p(x)+q(x))) \mathrm{d} x .
$$


The second integral on the right hand side of (3.5) is integrated by parts with respect to $t$, we get

$$
\begin{aligned}
& \int_{Q_{T}}\left(p^{\prime}(x+t)-q^{\prime}(x-t)\right)\left(\dot{v}(x, t)-\left(p^{\prime}(x+t)-q^{\prime}(x-t)\right)\right) \mathrm{d} x \mathrm{~d} t \\
= & -\int_{0}^{L}\left(p^{\prime}(x)-q^{\prime}(x)\right)(v(x, 0)-(p(x)+q(x))) \mathrm{d} x \\
& -\int_{Q_{T}}\left(p^{\prime \prime}(x+t)+q^{\prime \prime}(x-t)\right)(v(x, t)-(p(x+t)+q(x-t))) \mathrm{d} x \mathrm{~d} t,
\end{aligned}
$$

while the third one is integrated by parts with respect to $x$, we find

$$
\begin{aligned}
& \int_{Q_{T}}\left(p^{\prime}(x+t)+q^{\prime}(x-t)\right)\left(v^{\prime}(x, t)-\left(p^{\prime}(x+t)+q^{\prime}(x-t)\right)\right) \mathrm{d} x \mathrm{~d} t \\
= & -\int_{0}^{T}\left(p^{\prime}(t)+q^{\prime}(-t)\right)(v(0, t)-(p(t)+q(-t))) \mathrm{d} t \\
& -\int_{Q_{T}}\left(p^{\prime \prime}(x+t)+q^{\prime \prime}(x-t)\right)(v(x, t)-(p(x+t)+q(x-t))) \mathrm{d} x \mathrm{~d} t .
\end{aligned}
$$

We substitute (3.6)-(3.8) into (3.5) and according to (2.4), we find

$$
-\int_{0}^{T}\left(p^{\prime}(t)+q^{\prime}(-t)\right)(v(0, t)-(p(t)+q(-t))) \mathrm{d} t \geq 0,
$$

which implies that (1.4) holds.

Lemma 3.2. The weak solution (1.4) for $T=L$ define a solution to Cauchy problem (2.8).

Proof. Let $u$ be a solution to (1.4), it follows from Lemma 2.3 that $u$ has traces in $\mathrm{L}_{\text {loc }}^{2}(0, L)$ on $\{t=0\} \times(\epsilon, L)$ and it comes that $u^{0}=u(\cdot, 0)$ makes sense. We choose $\psi=v-u$ such that $\psi$ belongs to the space of infinitely differentiable functions on $Q_{T}$ with a compact support $\mathcal{D}\left(Q_{T}\right)$. Then it comes that

$$
-\int_{0}^{T}(\dot{u}, \dot{\psi}) \mathrm{d} t+\int_{0}^{T} a(u, \psi) \mathrm{d} t=0
$$

for all $\psi$ belonging to $\mathcal{D}\left(Q_{T}\right)$ where $a(u, \psi) \stackrel{\text { def }}{=} \int_{0}^{L} u^{\prime} \psi^{\prime} \mathrm{d} x \mathrm{~d} t$. This gives

$$
\langle\ddot{u}, \psi\rangle-\left\langle u^{\prime \prime}, \psi\right\rangle=0
$$

for all $\psi$ belonging to $\mathcal{D}\left(Q_{T}\right)$. Here the duality product between $\mathcal{D}^{\prime}\left(Q_{T}\right)$ and $\mathcal{D}\left(Q_{T}\right)$ is denoted by $\langle\cdot, \cdot\rangle$. Therefore, we may deduce that $\ddot{u}-u^{\prime \prime}$ vanishes in the sense of distributions in $Q_{T}$. Thus we have

$$
\int_{Q_{T}}\left(-\dot{u} \dot{\psi}+u^{\prime} \psi^{\prime}\right) \mathrm{d} x \mathrm{~d} t=0
$$

for all $\psi$ belonging to $\mathcal{D}\left(Q_{T}\right)$. We introduce the following notations: $\mu_{+} \stackrel{\text { def }}{=} x+t, \mu_{-} \stackrel{\text { def }}{=} x-t$ and $\Delta$ denotes the region bounded by the lines $\mu_{+}=-\mu_{-}, \mu_{+}=2 L-\mu_{-}, \mu_{-}=\mu_{+}$and $\mu_{-}=\mu_{+}-2 T$ in the plane $\left(\mu_{-}, \mu_{+}\right)$. Hence we have

$$
\dot{u}=\frac{\partial u}{\partial \mu_{+}}-\frac{\partial u}{\partial \mu_{-}}, \quad u^{\prime}=\frac{\partial u}{\partial \mu_{+}}+\frac{\partial u}{\partial \mu_{-}}, \quad \dot{\psi}=\frac{\partial \psi}{\partial \mu_{+}}-\frac{\partial \psi}{\partial \mu_{-}} \quad \text { and } \quad \psi^{\prime}=\frac{\partial \psi}{\partial \mu_{+}}+\frac{\partial \psi}{\partial \mu_{-}} .
$$


Carrying (3.10) into (3.9), we find

$$
-\int_{\Delta}\left(\frac{\partial u}{\partial \mu_{+}}-\frac{\partial u}{\partial \mu_{-}}\right)\left(\frac{\partial \psi}{\partial \mu_{+}}-\frac{\partial \psi}{\partial \mu_{-}}\right) \mathrm{d} \mu_{-} \mathrm{d} \mu_{+}+\int_{\Delta}\left(\frac{\partial u}{\partial \mu_{+}}+\frac{\partial u}{\partial \mu_{-}}\right)\left(\frac{\partial \psi}{\partial \mu_{+}}+\frac{\partial \psi}{\partial \mu_{-}}\right) \mathrm{d} \mu_{-} \mathrm{d} \mu_{+}=0
$$

for all $\psi$ belonging to $\mathcal{D}(\Delta)$. We observe that

$$
\int_{\Delta} \frac{\partial u}{\partial \mu_{+}} \frac{\partial \psi}{\partial \mu_{-}} \mathrm{d} \mu_{-} \mathrm{d} \mu_{+}+\int_{\Delta} \frac{\partial u}{\partial \mu_{-}} \frac{\partial \psi}{\partial \mu_{+}} \mathrm{d} \mu_{-} \mathrm{d} \mu_{+}=0
$$

for all $\psi$ belonging to $\mathcal{D}(\Delta)$. The first term in (3.11) is integrated by parts with respect to $\mu_{-}$while the second one is integrated by parts with respect to $\mu_{+}$to get

$$
-\left\langle\frac{\partial^{2} u}{\partial \mu_{+} \partial \mu_{-}}, \psi\right\rangle-\left\langle\frac{\partial^{2} u}{\partial \mu_{-} \partial \mu_{+}}, \psi\right\rangle=0
$$

for all $\psi$ belonging to $\mathcal{D}(\Delta)$. Since we have

$$
\left\langle\frac{\partial^{2} u}{\partial \mu_{+} \partial \mu_{-}}, \psi\right\rangle=\left\langle u, \frac{\partial^{2} \psi}{\partial \mu_{+} \partial \mu_{-}}\right\rangle=\left\langle\frac{\partial^{2} u}{\partial \mu_{-} \partial \mu_{+}}, \psi\right\rangle
$$

it follows that

$$
\left\langle\frac{\partial^{2} u}{\partial \mu_{+} \partial \mu_{-}}, \psi\right\rangle=0
$$

for all $\psi$ belonging to $\mathcal{D}(\Delta)$. Then we conclude that $\frac{\partial^{2} u}{\partial \mu_{+} \partial \mu_{-}}$vanishes which holds if and only if $u=p\left(\mu_{+}\right)+q\left(\mu_{-}\right)$.

We observe that Lemmas 2.3 and 2.4 imply that $\dot{u}(\cdot, 0)=v^{0}$ and $u^{\prime}(0, \cdot)$ belong to $H$ and to $\mathrm{L}^{2}(0, T-\epsilon)$, respectively. According to Theorem 5.1 (given in Appendix B), it comes that the following Green's formulas make sense

$$
\begin{aligned}
\int_{0}^{T} \int_{0}^{L}\left(\ddot{u}-u^{\prime \prime}\right) \psi \mathrm{d} x \mathrm{~d} t= & -\int_{0}^{T}((\dot{u}, \dot{\psi})-a(u, \psi)) \mathrm{d} t+\int_{0}^{T} u^{\prime}(L, t) \psi(L, t) \mathrm{d} t-\int_{0}^{T} u^{\prime}(0, t) \psi(0, t) \mathrm{d} t \\
& +(\dot{u}(x, T), \psi(x, T))-\left(v^{0}, \psi(x, 0)\right)
\end{aligned}
$$

for all $\psi$ belonging to $\mathbb{H}_{2}$. We insert (3.12) into (1.4) and we choose $v=u+\psi$, we obtain

$$
-\int_{0}^{T} u^{\prime}(0, t)(v(0, t)-u(0, t)) \mathrm{d} t \geq 0
$$

for all $v$ belonging to $\mathbb{K}$. Thanks to (2.1), we may deduce that (3.13) is equivalent to

$$
-\int_{0}^{T}\left(p^{\prime}(t)+q^{\prime}(-t)\right)(v(0, t)-(p(t)+q(-t))) \mathrm{d} t \geq 0
$$

for all $v$ belonging to $\mathbb{K}$. Since $p(t)+q(-t) \geq 0$, it follows that

$$
v(0, t)=p(t)+q(-t)+\alpha(t)
$$

for all $\alpha(t) \geq 0$. Therefore we may infer from (3.14) that

$$
-\int_{0}^{T}\left(p^{\prime}(t)+q^{\prime}(-t)\right) \alpha(t) \mathrm{d} t \geq 0
$$

for all $\alpha(t) \geq 0$, which implies that $p^{\prime}(t)+q^{\prime}(-t) \leq 0$ for almost every $t \in(0, T)$. Finally we choose $v(0, t)=0$ and $v(0, t)=2(p(t)+q(-t))$ in $(3.14)$, we get

$$
\int_{0}^{T}\left(p^{\prime}(t)+q^{\prime}(-t)\right)(p(t)+q(-t)) \mathrm{d} t=0
$$

which allows us to infer that $\left(p^{\prime}(t)+q^{\prime}(-t)\right)(p(t)+q(-t))$ vanishes for almost every $t \in(0, T)$. This concludes the proof. 


\section{Convergence of mass Redistribution method}

The semi-discretized problem by using finite elements is not well-posed which emphasizes some instabilities of time integration schemes (see $[16,23,24,26,27])$. In the literature many different approaches were elaborated to overcome this difficulty. For instance the uniqueness for an impact law of rigid bodies can be recovered by introducing a restitution coefficient (see [25]). However, this approach is not totally satisfactory for deformable bodies. Indeed the presence of oscillations due to displacement and to normal stress on the contact boundary induces some difficulties in the construction of energy conserving schemes (see [20,21,25]). Another approach consists in using the penalty method which introduces some oscillations as well but which can be reduced with a damping technique (see [33]). One of the key point to avoid oscillations is to use the mass redistribution method, the reader is referred to [16] and the references therein.

We first approximate (1.1)-(1.3) by using the Lagrange affine finite element method. To this aim, we define $h=\frac{L}{n}$ where $n$ is an integer and

$$
V^{h} \stackrel{\text { def }}{=}\left\{v^{h} \in \mathrm{C}^{0}([0, L]):\left.v^{h}\right|_{\left[a_{i}, a_{i+1}\right]} \in P_{1}, i=0, \ldots, n-1, v^{h}(L)=0\right\} .
$$

Here, $a_{i} \stackrel{\text { def }}{=} i h, i=0,1, \ldots, n$, and $P_{1}$ is the space of polynomials of degree less than or equal to 1 . A classical basis of $V^{h}$ is given by the sequence of shape functions $\varphi_{i} \in V^{h}$ for $i=0,1, \ldots, n-1$, defined by

$$
\varphi_{i}(x) \stackrel{\text { def }}{=} \begin{cases}1-\frac{\left|x-a_{i}\right|}{h} & \text { if } \quad x \in\left[a_{\max (i-1,0)}, a_{i+1}\right], \\ 0 & \text { otherwise. }\end{cases}
$$

Note that $\varphi_{i}\left(a_{j}\right)=\delta_{i j}, j=0,1, \ldots, n-1$, i.e. $\delta_{i j}=1$ if $i=j$ and $\delta$ vanishes otherwise $(\delta$ is the Kronecker symbol). We approximate the solution $u$ belonging to $V$ to the weak formulation (1.4) by

$$
u^{h}(x, t)=\sum_{j=0}^{n-1} u_{j}(t) \varphi_{j}(x) .
$$

Consequently, we have $u_{i}=u^{h}\left(a_{i}\right), i=0,1, \ldots, n-1$. The weak formulation (1.4) is approximated as follows

$$
\left(\mathrm{P}_{u^{h}}\right)\left\{\begin{array}{l}
\text { find } u^{h}:[0, T] \rightarrow V^{h} \text { and } \lambda:[0, T] \rightarrow \mathbb{R} \text { such that for all } v^{h} \in V^{h} \\
\int_{0}^{L} \ddot{u}^{h} v^{h} \mathrm{~d} x+a\left(u^{h}, v^{h}\right)=-\lambda v^{h}(0) \quad \text { a.e. } \quad t \in[0, T] \\
0 \leq u^{h}(0, \cdot) \perp \lambda \leq 0 \quad \text { a.e. } \quad t \in[0, T] \\
u^{h}(\cdot, 0)=u^{0 h} \quad \text { and } \quad \dot{u}^{h}(\cdot, 0)=v^{0 h}
\end{array}\right.
$$

where $u^{0 h}$ and $v^{0 h}$ belong to $V^{h}$ such that

$$
\lim _{h \rightarrow 0}\left(\left\|u^{0 h}-u^{0}\right\|_{V}+\left\|v^{0 h}-v^{0}\right\|_{H}\right)=0,
$$

where $\lambda$ is the Lagrange multiplier. We introduce now the following notations: $U \stackrel{\text { def }}{=}\left(u_{0}, \ldots, u_{n-1}\right)^{\top}$ and $e_{0} \stackrel{\text { def }}{=}$ $(1,0, \ldots, 0)^{\top}$. The corresponding algebraic formulation is given by

$$
\left(\mathrm{P}_{U \lambda}\right)\left\{\begin{array}{l}
\text { find } U:[0, T] \rightarrow \mathbb{R}^{n} \text { and } \lambda:[0, T] \rightarrow \mathbb{R} \text { such that } \\
M \ddot{U}+S U=-\lambda e_{0} \quad \text { a.e. } \quad t \in[0, T] \\
0 \leq u_{0} \perp \lambda \leq 0 \quad \text { a.e. } \quad t \in[0, T] \\
U(0)=U^{0} \quad \text { and } \quad \dot{U}(0)=V^{0}
\end{array}\right.
$$


where $M$ and $S$ denote the mass and stiffness matrices, respectively;

$$
M_{i j} \stackrel{\text { def }}{=} \int_{0}^{L} \varphi_{i} \varphi_{j} \mathrm{~d} x \quad \text { and } S_{i j} \stackrel{\text { def }}{=} a\left(\varphi_{i}, \varphi_{j}\right)=\frac{1}{h}\left(\begin{array}{rrrrrr}
1 & -1 & 0 & \cdots & \cdots & 0 \\
-1 & 2 & -1 & \ddots & & \vdots \\
0 & -1 & 2 & \ddots & \ddots & \vdots \\
\vdots & \ddots & \ddots & \ddots & \ddots & 0 \\
\vdots & & \ddots & -1 & 2 & -1 \\
0 & \cdots & \cdots & 0 & -1 & 1
\end{array}\right) \text {, }
$$

for all $i, j \in[0, n-1]$.

We define now the modified mass matrix as follows: $M_{i j}^{\text {mod }} \stackrel{\text { def }}{=} \int_{h}^{L} \varphi_{i} \varphi_{j} \mathrm{~d} x$. Clearly, we may observe that $M_{0 i}=M_{i 0}=0$ for all $i=0, \ldots, n-1$. Therefore, the modified mass matrix reads

$$
M^{\text {mod }} \stackrel{\text { def }}{=}\left(\begin{array}{ll}
0 & 0 \\
0 & \bar{M}
\end{array}\right) \text {. }
$$

Note that $\bar{M}_{i j}=M_{i+1, j+1}$ for all $i, j=1, \ldots, n-2$. We introduce now the following notations: $\bar{U} \stackrel{\text { def }}{=}$ $\left(u_{1}, \ldots, u_{n-1}\right)^{\top}$ and $\bar{S}_{i j} \stackrel{\text { def }}{=} S_{i+1, j+1}$ with $C \stackrel{\text { def }}{=} \int_{\Omega} \varphi_{i+1}^{\prime} \varphi_{0}^{\prime} \mathrm{d} x, i=0, \ldots, n-2$. Observe that $C=\left(S_{10}, 0, \ldots, 0\right)^{\top}$. Thus by using the above notations, we have

$$
S=\left(\begin{array}{cc}
S_{00} & C^{\top} \\
C & \bar{S}
\end{array}\right) \quad \text { and } \quad U=\left(\begin{array}{c}
u_{0} \\
\bar{U}
\end{array}\right) .
$$

This leads to an algebraic formulation of the semi-discrete approximation with mass redistribution method given by

$$
\left(\mathrm{P}_{U \lambda}^{\bmod }\right)\left\{\begin{array}{l}
\text { find } U:[0, T] \rightarrow \mathbb{R}^{n} \text { and } \lambda:[0, T] \rightarrow \mathbb{R} \text { such that } \\
\bar{M} \ddot{\bar{U}}+\bar{S} \bar{U}=-C u_{0} \quad \text { a.e. } \quad t \in[0, T] \\
S_{00} u_{0}+C^{\mathrm{\top}} \bar{U}=-\lambda \quad \text { a.e. } \quad t \in[0, T] \\
0 \leq u_{0} \perp \lambda \leq 0 \quad \text { a.e. } \quad t \in[0, T] \\
U(0)=U^{0} \quad \text { and } \quad \dot{U}(0)=V^{0}
\end{array}\right.
$$

It follows that

$$
u_{0}=\left(\frac{-\lambda-C^{\top} \bar{U}}{S_{00}}\right)=\left(\frac{-\lambda-S_{10} u_{1}}{S_{00}}\right)
$$

If $S_{10} u_{1} \geq 0$ then the compatibility condition gives $u_{0}=0$, so $\lambda=\left(C^{\top} \bar{U}\right)^{-}$otherwise we have $\lambda=0$. This implies that $u_{0}=\left(\frac{-S_{10} u_{1}}{S_{00}}\right)^{+}$, and then we may conclude that $\left(\mathrm{P}_{U \lambda}^{\bmod }\right)$ is equivalent to the following second order Lipschitz continuous ordinary differential equation:

$$
\left(\mathrm{P}_{\bar{U}}^{\bmod }\right)\left\{\begin{array}{l}
\text { find } \bar{U}:[0, T] \rightarrow \mathbb{R}^{n-1} \text { such that } \\
\bar{M} \ddot{\bar{U}}+\bar{S} \bar{U}=-C\left(\frac{-S_{10} u_{1}}{S_{00}}\right)^{+} \quad \text { a.e. } \quad t \in[0, T] \\
U(0)=U^{0} \quad \text { and } \quad \dot{U}(0)=V^{0}
\end{array}\right.
$$

Lemma 4.1 (existence and uniqueness results for $\left.\left(\mathrm{P}_{U \lambda}^{\bmod }\right)\right)$. Problem $\left(\mathrm{P}_{U \lambda}^{\bmod }\right)$ admits an unique solution $(U, \lambda)$ which is Lipschitz continuous. 
Proof. We use the fact that $\bar{M}$ is not a singular matrix as well as the same techniques detailed in [7] to establish that $\left(\mathrm{P}_{\bar{U}}^{\text {mod }}\right)$ possesses a unique Lipschitz continuous solution. On the other hand, we may deduce from $\left(\mathrm{P}_{U \lambda}^{\bmod }\right)$ that $u_{0}=\left(\frac{-S_{10} u_{1}}{S_{00}}\right)^{+}$and $\lambda=\left(C^{\top} \bar{U}\right)^{-}$. This allows us to conclude that $u_{0}$ and $\lambda$ are also Lipschitz continuous and then the conclusion is clear.

We deal with the energy balance and we establish the energy conservation of the solution to problem $\left(\mathrm{P}_{U \lambda}^{\bmod }\right)$. More precisely, the discrete energy associated to problem $\left(\mathrm{P}_{U \lambda}^{\bmod }\right)$ is given by

$$
\mathcal{E}(t)=\frac{1}{2}\left(\dot{U}^{\top} M^{\bmod } \dot{U}+U^{\top} S U\right)(t) .
$$

Lemma 4.2. The solution $(U, \lambda)$ to problem $\left(\mathrm{P}_{U \lambda}^{\bmod }\right)$ is energy conserving.

Proof. We observe first that

$$
\dot{U}^{\top} M^{\bmod } \ddot{U}+\dot{U}^{\top} S U=-\dot{U}^{\top} \lambda e_{0}
$$

Therefore, we integrate this expression over $(0, t)$ to get

$$
\forall t \in[0, T]: \mathcal{E}(t)-\mathcal{E}(0)=-\int_{0}^{t} \dot{u}_{0}(s) \lambda(s) \mathrm{d} s .
$$

Let us define $\omega \stackrel{\text { def }}{=}\left\{t \in[0, T]: u_{0}(t)>0\right\}$. On the one hand, the contact conditions imply that $\lambda=0$ on $\omega$. On the other hand, the continuity of $\lambda$ on $[0, T]$ gives that $\lambda=0$ on $\bar{\omega}$ where $\bar{\omega}$ is the closure to $\omega$. Furthermore, $\dot{u}_{0}$ vanishes in the interior of the set $[0, T] \backslash \omega$. Hence $\dot{u}_{0} \lambda=0$ on $[0, T]$ and we conclude that $\mathcal{E}(t)=\mathcal{E}(0)$ for all $t \in[0, T]$.

We observe that $\left(\mathrm{P}_{U \lambda}^{\bmod }\right)$ is equivalent to

$$
\left(\mathrm{P}_{u^{h}}^{\bmod }\right)\left\{\begin{array}{l}
\text { find } u^{h}:[0, T] \rightarrow V^{h} \text { such that for all } v^{h} \in K^{h} \\
\int_{0}^{T}\left(\int_{h}^{L} \ddot{u}^{h}\left(v^{h}-u^{h}\right) \mathrm{d} x+a\left(u^{h}, v^{h}-u^{h}\right)\right) \mathrm{d} t \geq 0 \\
u^{h}(\cdot, 0)=u^{0 h} \quad \text { and } \quad \dot{u}^{h}(\cdot, 0)=v^{0 h}
\end{array}\right.
$$

We establish below the convergence of the solution $u^{h}$ of $\left(\mathrm{P}_{u^{h}}^{\bmod }\right)$ to the solution of (1.4) by using some ideas developed in [32].

Theorem 4.3. Assume that (4.1) holds. Then, the solution $u^{h}$ of $\left(\mathrm{P}_{u^{h}}^{\bmod }\right)$ converges strongly in $\mathbb{H}_{2}$ to the unique solution of (1.4) as $h$ tends to 0 .

Proof. We observe that

$$
\int_{0}^{L}\left|\dot{u}^{h}(x, t)\right|^{2} \mathrm{~d} x=\int_{0}^{h}\left|\dot{u}^{h}(x, t)\right|^{2} \mathrm{~d} x+\int_{h}^{L}\left|\dot{u}^{h}(x, t)\right|^{2} \mathrm{~d} x .
$$

We evaluate now the right hand side of (4.3). To this aim, we note that $u_{0}(t)=-\frac{S_{10}}{S_{00}}\left(u_{1}(t)\right)^{+}$implies that

$$
\left|\dot{u}_{0}(t)\right| \leq\left|\dot{u}_{1}(t)\right|,
$$

since $-\frac{S_{10}}{S_{00}}=1$. Therefore, by using (4.4) and Cauchy Schwarz's inequality, we may deduce that there exists $C_{0}>0$ such that

$$
\int_{0}^{h}\left|\dot{u}^{h}(x, t)\right|^{2} \mathrm{~d} x=\int_{0}^{h}\left|\dot{u}_{1}(t) \varphi_{1}(x)+\dot{u}_{0}(t) \varphi_{0}(x)\right|^{2} \mathrm{~d} x \leq C_{0} h\left|\dot{u}_{1}(t)\right|^{2}
$$


Furthermore, the energy conservation of Lemma 4.2 implies that there exists $C_{1}>0$ such that

$$
\int_{h}^{L}\left|\dot{u}^{h}(x, t)\right|^{2} \mathrm{~d} x \leq C_{1}
$$

Consequently, we deduce that

$$
\int_{i h}^{(i+1) h}\left|\dot{u}^{h}(x, t)\right|^{2} \mathrm{~d} x=\int_{i h}^{(i+1) h}\left|\dot{u}_{i+1}(t) \varphi_{i+1}(x)+\dot{u}_{i}(t) \varphi_{i}(x)\right|^{2} \mathrm{~d} x \leq C_{1}
$$

for $i=1, \ldots, n-1$. We conclude that for sufficiently small $h$, we get

$$
\left|\dot{u}_{i+1}(t)\right|^{2}+\left|\dot{u}_{i}(t)\right|^{2} \leq \frac{6 C_{1}}{h}
$$

for $i=1, \ldots, n-1$. Therefore $\left|\dot{u}_{1}(t)\right|^{2} \leq \frac{6 C_{1}}{h}$ which implies that $\left\|\dot{u}^{h}(\cdot, t)\right\|_{\mathrm{L}^{2}(0, h)}$ is bounded independently of $h$ and then $\left\|\dot{u}^{h}(\cdot, t)\right\|_{H}$ is also bounded independently of $h$. By using Lemma 4.2 , we can prove that $\left\|u^{h}\right\|_{V}$ is bounded. It follows that there exists $C>0$ independent of $t$ such that

$$
\sup _{t \in[0, T]}\left(\left\|u^{h}(\cdot, t)\right\|_{V}+\left\|\dot{u}^{h}(\cdot, t)\right\|_{H}\right) \leq C .
$$

Then, it is possible to extract a subsequence, still denoted by $u^{h}$, such that

$$
\begin{aligned}
& u^{h} \rightarrow u \quad \text { in } \quad \mathrm{L}^{\infty}(0, T ; V) \quad \text { weak } \quad *, \\
& \dot{u}^{h} \rightarrow \dot{u} \quad \text { in } \quad \mathrm{L}^{\infty}(0, T ; H) \quad \text { weak } \quad * .
\end{aligned}
$$

Let us define

$$
\mathbb{H}_{\infty} \stackrel{\text { def }}{=}\left\{u \in \mathrm{L}^{\infty}(0, T ; V): \dot{u} \in \mathrm{L}^{\infty}(0, T ; H)\right\}
$$

endowed with the following norm

$$
\|u\|_{\mathbb{H}_{\infty}} \stackrel{\text { def }}{=} \operatorname{ess~sup}_{t \in[0, T]}\left(\|u(\cdot, t)\|_{V}+\|\dot{u}(\cdot, t)\|_{H}\right) .
$$

We may infer from (4.5) that

$$
u^{h} \rightarrow u \quad \text { in } \quad \mathbb{H}_{\infty} \quad \text { weak } \quad * .
$$

Since for all $\alpha<\frac{1}{2}$ the following injections $\mathbb{H}_{\infty} \hookrightarrow \mathrm{C}^{0, \frac{1}{2}}\left(Q_{T}\right) \hookrightarrow \hookrightarrow \mathrm{C}^{0, \alpha}\left(Q_{T}\right)$ hold (see [32]), where $\hookrightarrow$ is continuous embedding and $\hookrightarrow \hookrightarrow$ is compact embedding, we get

$$
u^{h} \rightarrow u \quad \text { in } \quad \mathrm{C}^{0, \alpha}\left(Q_{T}\right)
$$

for all $\alpha<\frac{1}{2}$. We observe that both $u^{h}(t)$ and $u(t)$ belong to $\mathbb{K}$.

In order to prove that the limit $u$ satisfies (1.4), it is necessary to choose convenient test functions. We approximate the elements of $\mathbb{K}$ before projecting them onto $V^{h}$. Indeed, the $\mathrm{L}^{2}$ projection does not conserve the constraint at $x=0$, and therefore, the elements of $\mathbb{K}$ need another approximation in order to satisfy the constraint strictly. To this aim, let $v$ be an element of $\mathbb{K}$ which is equal to $u$ for $t \geq T-\epsilon$ and let

$$
v^{\beta}(x, t) \stackrel{\text { def }}{=} \begin{cases}u(x, t)+\frac{1}{\beta} \int_{t}^{t+\beta}(v(x, s)-u(x, s)) \mathrm{d} s+k(\beta)(L-x) g(t) & \text { if } \quad t \leq T-\beta, \\ u(x, t) & \text { if } \quad t \geq T-\beta,\end{cases}
$$

for all $\beta \leq \frac{\epsilon}{4}$. Here $g(t)$ is smooth, positive function (see Fig. 3) and satisfying 


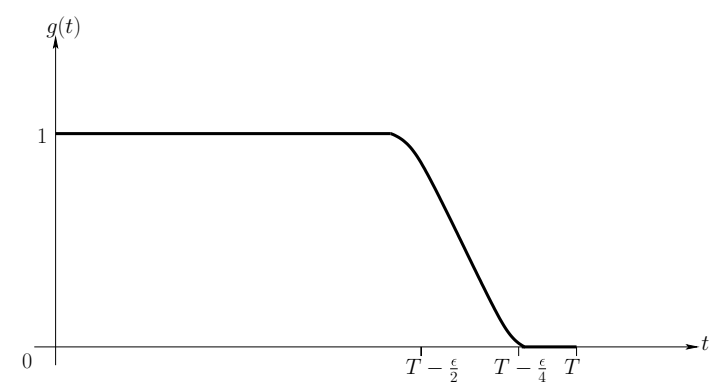

Figure 3. Smooth and positive function $g(t)$.

$$
g(t) \stackrel{\text { def }}{=}\left\{\begin{array}{lll}
1 & \text { if } & t \in\left[0, T-\frac{\epsilon}{2}\right], \\
0 & \text { if } & t \in\left[T-\frac{\epsilon}{4}, T\right] .
\end{array}\right.
$$

The next step consists to choose adequately $k(\beta)$. Since $u$ belongs to $C^{0, \frac{1}{2}}\left(Q_{T}\right)$, we may deduce that there exists $C>0$, such that

$$
\begin{aligned}
\left|u(0, t)-\beta^{-1} \int_{t}^{t+\beta} u(0, s) \mathrm{d} s\right| & \leq \beta^{-1} \int_{t}^{t+\beta}|u(0, t)-u(0, s)| \mathrm{d} s \\
& \leq C\|u\|_{\mathbb{H}_{\infty}} \beta^{-1} \int_{0}^{\beta} \sqrt{s} \mathrm{~d} s=\frac{2}{3} C\|u\|_{\mathbb{H}_{\infty}} \sqrt{\beta} .
\end{aligned}
$$

Furthermore, we have the following inequality

$$
v^{\beta}(0, t) \geq \beta^{-1} \int_{t}^{t+\beta} v(0, s) \mathrm{d} s-\frac{2}{3} C\|u\|_{\mathbb{H}_{\infty}} \sqrt{\beta}+k(\beta) L g(t)
$$

for all $t \leq T-\frac{\epsilon}{2}$. Choosing $k(\beta)=\frac{5}{3 L} C\|u\|_{\mathbb{H}_{\infty}} \sqrt{\beta}$, we get

$$
v^{\beta}(0, t) \geq C\|u\|_{\mathbb{H}_{\infty}} \sqrt{\beta}
$$

for all $t \leq T-\frac{\epsilon}{2}$. On the other hand, we have

$$
v^{\beta}(x, t)=u(x, t)+k(\beta)(L-x) g(t)
$$

for all $t$ belonging to $\left[T-\frac{\epsilon}{2}, T-\beta\right]$. Hence $v^{\beta}$ belongs to $\mathbb{K}$ and in the other hand $v^{\beta}$ belongs to $\mathrm{L}^{\infty}(0, T ; V)$ because

$$
\left\|v^{\beta}(\cdot, t)-u(\cdot, t)\right\|_{V} \leq k(\beta) L g(t)+\frac{1}{\sqrt{\beta}}\|v-u\|_{\mathbb{H}_{2}} .
$$

We denote by $Q^{h}$ the orthogonal projection onto $V^{h}$ with respect to the scalar product in $H$ such that $\left\|Q^{h} z-z\right\|_{V} \rightarrow 0$ when $h \rightarrow 0$ for all $z \in V$ (see [12]). The Sobolev injections imply that there exists a sequence $\alpha_{h}$ converging to zero as $h$ tends to zero such that

$$
\left\|Q^{h} z-z\right\|_{\mathrm{C}^{0}} \leq \alpha_{h}\|z\|_{V}
$$

for all $z$ belonging to $V$ with $\lim _{h \rightarrow 0} \alpha_{h}=0$. The test function is defined as follows:

$$
v^{h}(\cdot, t) \stackrel{\text { def }}{=} u^{h}(\cdot, t)+Q^{h}\left(v^{\beta}-u\right)(\cdot, t)
$$


for all $t$ belonging to $[0, T]$. By using a continuity argument, $v^{h}(0, \cdot) \geq 0$ for $h$ small enough. Carrying (4.6) into $\left(\mathrm{P}_{u^{h}}^{\bmod }\right)$ and using the integration by parts, we find

$$
\begin{aligned}
& -\int_{0}^{L} \dot{u}^{h}(\cdot, 0) Q^{h}\left(v^{\beta}-u\right)(\cdot, 0) \mathrm{d} x+\int_{0}^{h} \dot{u}^{h}(\cdot, 0) Q^{h}\left(v^{\beta}-u\right)(\cdot, 0) \mathrm{d} x-\int_{0}^{T} \int_{0}^{L} \dot{u}^{h}(\cdot, t) Q^{h}\left(\dot{v}^{\beta}-\dot{u}\right)(\cdot, t) \mathrm{d} x \mathrm{~d} t \\
& +\int_{0}^{T} \int_{0}^{h} \dot{u}^{h}(\cdot, t) Q^{h}\left(\dot{v}^{\beta}-\dot{u}\right)(\cdot, t) \mathrm{d} x \mathrm{~d} t+\int_{0}^{T} a\left(u^{h}(\cdot, t), Q^{h}\left(v^{\beta}-u\right)(\cdot, t) \mathrm{d} t \geq 0 .\right.
\end{aligned}
$$

Since $\left(v^{\beta}-u\right)(\cdot, t)$ is bounded in $\mathbb{H}_{\infty}$, the above integration makes sense. Thus we may pass to the limit when $h$ tends to zero. Since we have

$$
Q^{h}\left(\dot{v}^{\beta}-\dot{u}\right) \rightarrow\left(\dot{v}^{\beta}-\dot{u}\right) \quad \text { in } \mathrm{L}^{2}(0, T ; H) \quad \text { and } \quad Q^{h}\left(v^{\beta}-u\right) \rightarrow\left(v^{\beta}-u\right) \quad \text { in } \mathrm{L}^{2}(0, T ; V) .
$$

Then, we conclude that

$$
-\int_{0}^{L} \dot{v}^{0}\left(v^{\beta}-u\right)(\cdot, 0) \mathrm{d} x-\int_{0}^{L} \int_{0}^{T} \dot{u}(\cdot, t)\left(\dot{v}^{\beta}-\dot{u}\right)(\cdot, t) \mathrm{d} t \mathrm{~d} x+\int_{0}^{T} a\left(u(\cdot, t),\left(v^{\beta}-u\right)(\cdot, t)\right) \mathrm{d} t \geq 0 .
$$

We pass now to the limit with respect to $\beta$ so we obtain variational formulation (1.4).

On the one hand, we observe that Lemma 2.5 leads to

$$
\int_{0}^{L}\left(|\dot{u}(\cdot, t)|^{2}+\left|u^{\prime}(\cdot, t)\right|^{2}\right) \mathrm{d} x=\int_{0}^{L}\left(\left|v^{0}\right|^{2}+\left|u^{0^{\prime}}\right|^{2}\right) \mathrm{d} x .
$$

On the other hand, Lemma 4.2 implies that

$$
\int_{h}^{L}\left|\dot{u}^{h}(\cdot, t)\right|^{2} \mathrm{~d} x+a\left(u^{h}(\cdot, t), u^{h}(\cdot, t)\right)=\int_{h}^{L}\left|\dot{u}^{h}(\cdot, 0)\right|^{2} \mathrm{~d} x+a\left(u^{h}(\cdot, 0), u^{h}(\cdot, 0)\right),
$$

which by using (4.1) and (4.5) gives

$$
\lim _{h \rightarrow 0} \int_{h}^{L}\left(\left|\dot{u}^{h}(\cdot, t)\right|^{2}+\left|u^{h^{\prime}}(\cdot, t)\right|^{2}\right) \mathrm{d} x=\int_{h}^{L}\left(\left|v^{0}\right|^{2}+\left|u^{0^{\prime}}\right|^{2}\right) \mathrm{d} x .
$$

Therefore from (4.7) and (4.8), it comes that

$$
\lim _{h \rightarrow 0} \int_{h}^{L}\left(\left|\dot{u}^{h}(\cdot, t)\right|^{2}+\left|u^{h^{\prime}}(\cdot, t)\right|^{2}\right) \mathrm{d} x=\int_{0}^{L}\left(|\dot{u}(\cdot, t)|^{2}+\left|u^{\prime}(\cdot, t)\right|^{2}\right) \mathrm{d} x .
$$

Since $u^{h}$ converges weakly to $u$ in $\mathbb{H}_{\infty}$ and $\left\|u^{h}\right\|_{\mathbb{H}_{\infty}}$ converges to $\|u\|_{\mathbb{H}_{\infty}}$ and since $\mathbb{H}_{\infty} \hookrightarrow \mathbb{H}_{2}$ then we conclude that $u^{h}$ converges strongly to $u$ in $\mathbb{H}_{2}$.

\section{NumERICAL EXAMPLES}

We perform a finite element discretization in space and we use a classical Newmark time stepping method. This leads to consider the following problem:

$$
\left\{\begin{array}{l}
U^{n+1}=U^{n}+\Delta t \dot{U}^{n}+\left(\frac{1}{2}-\beta\right) \Delta t^{2} \ddot{U}^{n}+\beta \Delta t^{2} \ddot{U}^{n+1} \\
\dot{U}^{n+1}=\dot{U}^{n}+(1-\gamma) \Delta t \ddot{U}^{n}+\gamma \Delta t \ddot{U}^{n+1} \\
M \ddot{U}^{n+1}+S U^{n+1}=-\lambda^{n+1} e_{0} \\
0 \leq u_{0}^{n+1} \perp \lambda^{n+1} \leq 0 \\
U(0)=U^{0} \quad \text { and } \quad \dot{U}(0)=V^{0}
\end{array}\right.
$$



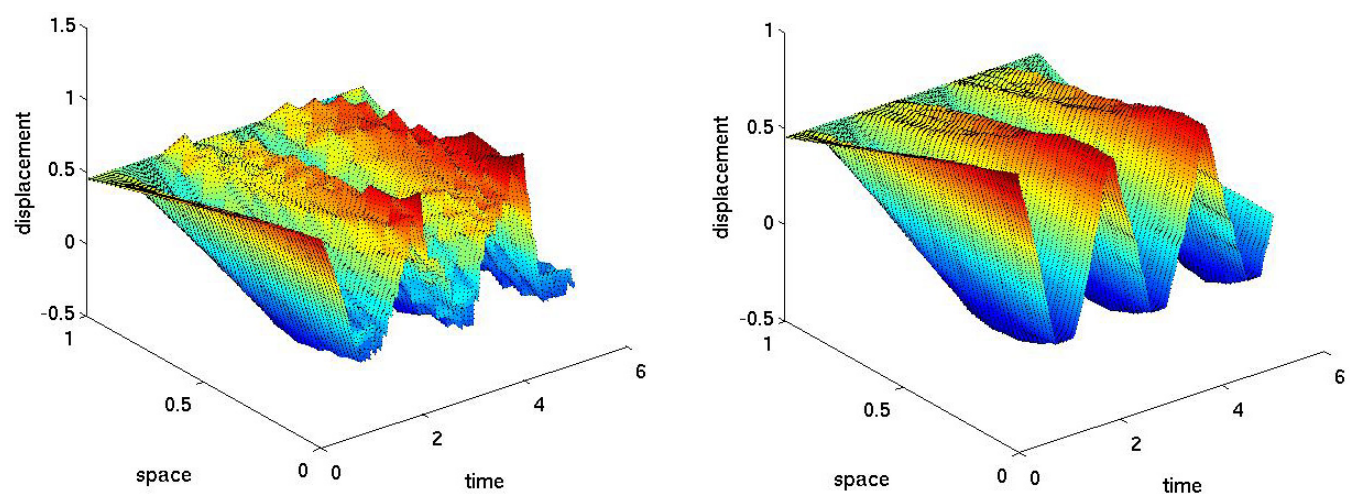

Figure 4. Numerical experiments with standard mass matrix (left) and with modified mass matrix (right).
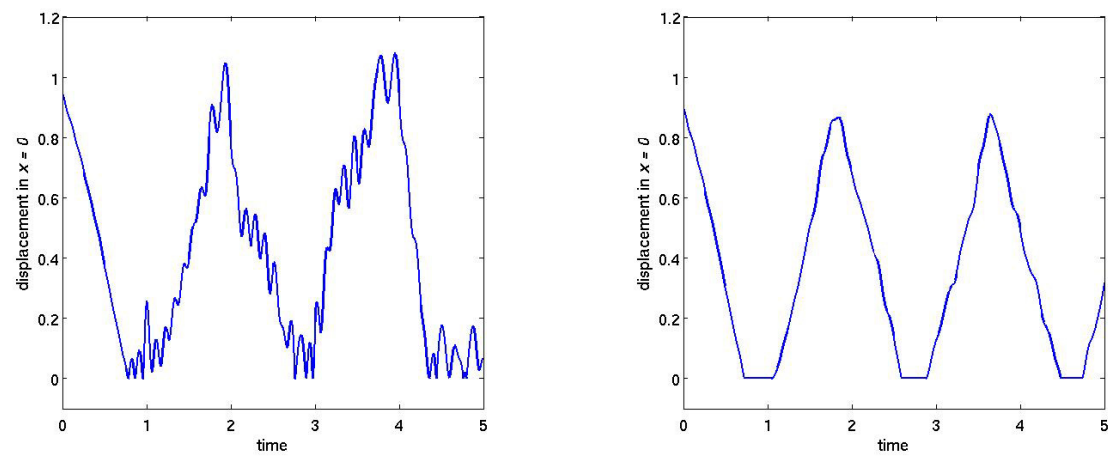

FiguRE 5. Numerical experiments with standard mass matrix (left) and with modified mass matrix (right) in the contact point $x=0$.

where $\beta \in] 0,1 / 2[$ and $\gamma \in] 1 / 2,1[$ are the classical parameters of the Newmark scheme. Note that if $\beta=0.25$ and $\gamma=0.5$, the scheme (5.1) is the so-called Crank-Nicholson scheme which is an implicit, unconditionally stable and second-order accurate scheme for elastodynamic problems without contact conditions and moreover it is energy conserving (see [16]). On the other hand, it is well known that the space-semi discretization of contact problems in elastodynamics present some numerical instabilities (see [15]) which can be avoided by using a modified mass method (see [16] and the references therein). We make below some comparisons between two different approaches; the one using a standard mass matrix and the one using a modified mass matrix. The parameters used in the numerical simulations are the space step $\Delta x=0.1$, the time step $\Delta t=0.01$, the initial displacement $u^{0}(x)=0.5 x-0.5$, the initial velocity $v^{0}(x)=0$ and the Dirichlet value $u(L, t)=0.45$ with $L=1$ and $T=5$. The numerical experiments are performed by employing the finite element library Getfem ++ (see [29]). In particular, the generalized Newton algorithm has been used to compute the unique solution of $(5.1)$ (see $[1,28]$ ). The numerical results show that when the constraint is active, small oscillations occur in the case where $M$ is a standard mass matrix (see Figs. 4 and 5 (left)) while these oscillations do not exist in the case where $M$ is a modified matrix (see Figs. 4 and 5 (right)). Furthermore, we can observe in Figure 6 (left), the energy is increasing with the standard mass matrix while with modified mass matrix (right), it is almost conservative. 

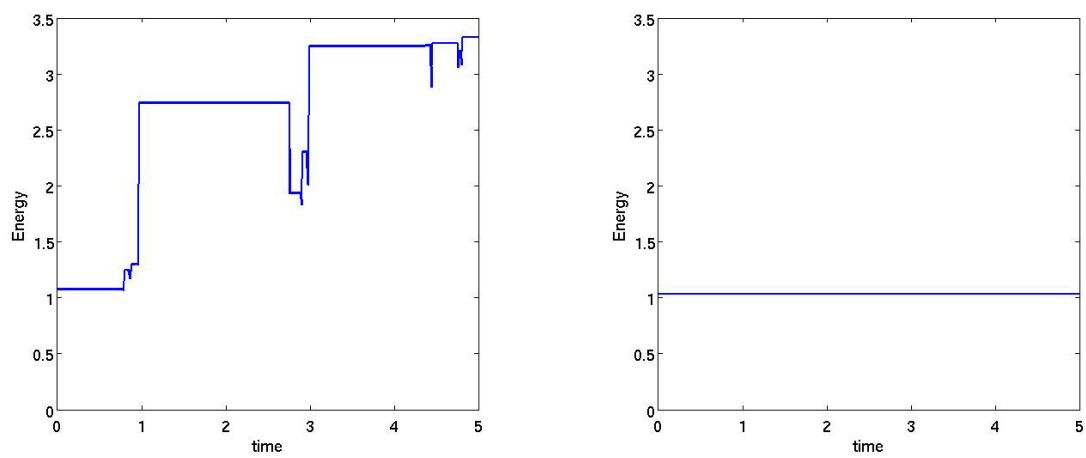

FiguRE 6. Energy evolution for standard mass matrix (left) and for modified mass matrix (right).

We present now some numerical results obtained for an undeformed elastic bar which is dropped with some initial velocity on a rigid obstacle and we compare the exact solution to the approximated one obtained by using the mass redistribution method. More precisely, we assume that this bar fall from a height $u^{0}$, with an initial velocity $-v^{0}$ and under the gravity $g \geq 0$. Furthermore the both ends of the bar are free to move, as long as the bar does not hit a rigid obstacle. The length and the Young modulus of the bar are denoted by $L$ and $E$, respectively. Let $u(x, t)$ be the displacement at time $t$ of the material point of spatial coordinate $x \in[0, L]$ and the contact pressure equal to the normal stress $-E u^{\prime}(0, \cdot)$. Then the mathematical problem can be formulated as follows:

$$
\ddot{u}(x, t)-E u^{\prime \prime}(x, t)=-\left(g+E u^{\prime}(0, t)\right), \quad(x, t) \in(0, L) \times(0, T),
$$

with Cauchy initial data

$$
u(\cdot, 0)=u^{0} \quad \text { and } \quad \dot{u}(\cdot, 0)=-v^{0},
$$

and Signorini and Neumann boundary conditions at $x=0$ and $x=L$, respectively, for $t>0$

$$
0 \leq u(0, \cdot) \perp E u^{\prime}(0, \cdot) \leq 0 \quad \text { and } \quad u^{\prime}(L, \cdot)=0 .
$$

The existence and uniqueness results for (5.2)-(5.4) is obtained by rewriting this problem as a differential inclusion problem and then by using the same techniques detailed in the proof of Theorem 2.1. Since it is quite a routine to adapt this proof to the case considered here, we let the verification to the reader. In order to calculate the analytical solution to problem (5.2)-(5.4), we distinguish three phases, namely before the contact, during the contact and after the contact. To this aim, we choose $v^{0}=0$ and $g>0$ so that the bar can make several impact. The bar reaches the rigid obstacle at time $t_{1}=\sqrt{\frac{2 u^{0}}{g}}$ with the velocity equal to $\sqrt{2 u^{0} g}$. After the impact, the bar stays in contact with the rigid body as soon as a shock wave travels from bottom of the bar to the top and vice versa then the bar takes off. The wave takes a time $\frac{L}{\sqrt{E}}$ to travel along the bar. The impacts occur at time $t_{4 k+1}=3 \frac{L}{\sqrt{E}}+16 k \frac{L}{\sqrt{E}}, t_{4 k+2}=t_{4 k+1}+2 \frac{L}{\sqrt{E}}, t_{4 k+3}=t_{4 k+1}+8 \frac{L}{\sqrt{E}}, t_{4 k+4}=t_{4 k+1}+10 \frac{L}{\sqrt{E}}$. We introduce also the following notations:

$$
\begin{aligned}
& h_{1}(x, t)=-\sqrt{\frac{2 u^{0}}{g}} \min \left(\frac{x}{\sqrt{E}}, \frac{L}{\sqrt{E}}-\left|t-\frac{L}{\sqrt{E}}\right|\right)+\sum_{n=1}^{\infty} \frac{2 g}{E L \nu_{n}^{3}}\left(\cos \left(\sqrt{E} \nu_{n} t\right)-1\right) \sin \left(\nu_{n} x\right), \\
& h_{2}(x, t)=u^{0}-\frac{1}{2} g\left(t-\sqrt{\frac{2 u^{0}}{g}}\right)^{2}-\frac{2 g L^{2}}{3 E}+\sum_{n=1}^{\infty} \frac{4 g}{E \lambda_{n}^{2}} \cos \left(\sqrt{E} \lambda_{n} t\right) \cos \left(\lambda_{n} x\right),
\end{aligned}
$$



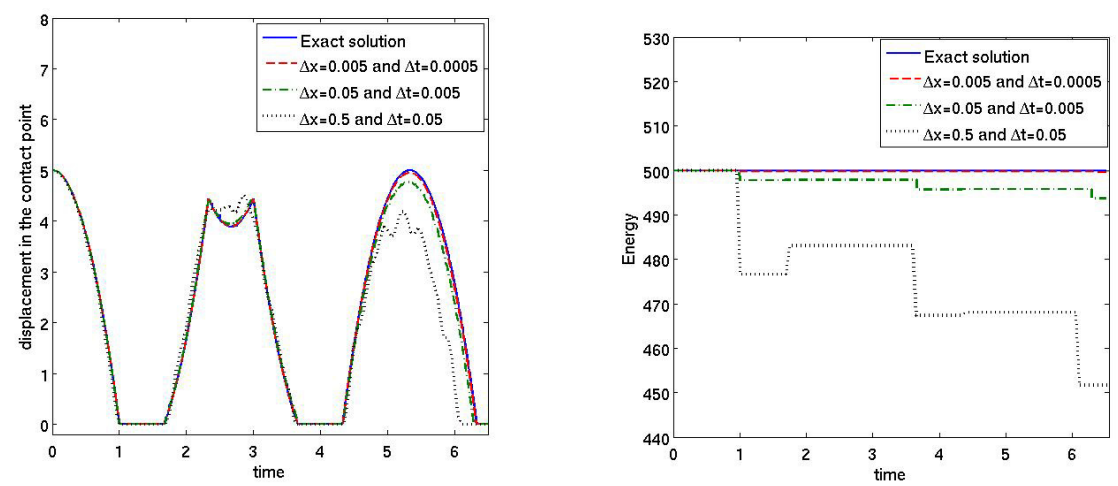

FiguRE 7. Numerical convergence of the solution associated to problem with mass redistribution method to the exact solution in the contact point $x=0$ (left). Numerical convergence of the energy evolution associated to mass redistribution method to the exact energy (right).

with $\nu_{n}=\left(n-\frac{1}{2}\right) \frac{\pi}{L}$ and $\lambda_{n}=n \frac{\pi}{L}$. Then, the explicit solution reads as

$$
u(x, t)=\left\{\begin{array}{lll}
u^{0}-\frac{1}{2} g t^{2} & \text { if } \quad t \leq t_{1}, \\
h_{1}\left(x, t-t_{4 k+1}\right) & \text { if } \quad t_{4 k+1}<t \leq t_{4 k+2}, \\
h_{2}\left(x, t-t_{4 k+2}\right) & \text { if } \quad t_{4 k+2}<t \leq t_{4 k+3}, \\
h_{1}\left(x, t_{4 k+4}-t\right) & \text { if } \quad t_{4 k+3}<t \leq t_{4 k+4}, \\
u^{0}-\frac{1}{2} g\left(t-t_{4 k+4}-\sqrt{\frac{2 u^{0}}{g}}\right)^{2} & \text { if } \quad t_{4 k+4}<t \leq t_{4(k+1)+1} .
\end{array}\right.
$$

Here, some details are omitted, the reader is referred to [11,35] for a detailed explanations. We choose $L=10$, $T=6.5, E=900, g=10$, the initial data $u^{0}(x)=5, v^{0}(x)=0$ and Neumann value $u^{\prime}(L, t)=0$. The Newmark time stepping method with $\beta=0.25$ and $\gamma=0.5$ is used to evaluate the approximated solution. Let us emphasize that if the space step $\Delta x$ and time step $\Delta t$ tend to 0 , the approximated solution obtained by using the mass redistribution method $\left(\mathrm{P}_{U \lambda}^{\bmod }\right)$ converges to the solution of (5.2)-(5.4) explicitly given by $(5.5)$ (see Fig. 7 (left)). On the other hand, we can write at least formally an energy relation for (5.2): we multiply this equation by $\dot{u}$, we integrate by parts over $Q_{\tau}, \tau \in[0, T]$, we get

$$
\frac{1}{2} \int_{0}^{L}|\dot{u}(\cdot, \tau)|^{2} \mathrm{~d} x+\frac{1}{2} \int_{0}^{L}\left|\sqrt{E} u^{\prime}(\cdot, \tau)\right|^{2} \mathrm{~d} x=-\int_{Q_{\tau}} g \dot{u} \mathrm{~d} x \mathrm{~d} t
$$

for all $\tau$ belonging to $[0, T]$. Observe that the energy tends to be conserved when the space step $\Delta x$ and the time step $\Delta t$ tend to 0 and the energy decreases otherwise (see Fig. 7 (right)).

\section{APPENDix A}

The aim of this section is to give the proofs of Theorem 2.1 and Lemma 2.4. Furthermore, a regularity result is also presented. Notice that Theorem 2.1 is a straightforward application of ([9], p. 59, Cor. 5.4).

Proof of Theorem 2.1. We verify the assumptions of ([9], p. 59, Cor. 5.4). We define $F(t, f(t)) \stackrel{\text { def }}{=}-J_{N}(f(t))-$ $2 p^{\prime}(t)$. Hence we choose $f(t)=y$ which gives that

$$
F(t, y)=-J_{N}(y)-2 p^{\prime}(t)
$$


The multivalued map $F:[0, L] \times(-\infty, 0] \rightarrow \mathcal{P}(\overline{\mathbb{R}}) \backslash \emptyset$ has closed convex values and $F$ is measurable with respect to its second variable. We prove now that $F$ is upper semi-continuous with respect to its second variable which is equivalent to establish that $J_{N}$ is upper semi-continuous. Note that if $A \subset \overline{\mathbb{R}}, J_{N}^{-1}(A)=[0,+\infty)$ or $J_{N}^{-1}(A)=\{0\}$ or $J_{N}^{-1}(A)=\emptyset$ which are closed sets. According to Definition [9], p. 4, Definition 1.1, $J_{N}$ is upper semi-continuous.

We verify that there is a function $r(t, y)=c(t)(1+|y|)$ with $c \in \mathrm{L}^{1}(0, L)$ such that

$$
F(t, y) \cap r(t, y) \bar{B}_{1}(0) \cap T_{(-\infty, 0]}(y) \neq \emptyset \quad \text { on } \quad[0, L] \times(-\infty, 0],
$$

where $B_{1}(0)$ is the ball of radius 1 at the origin and $T_{(-\infty, 0]}(y)$ is the tangent cone on $y$, the reader is referred to [9] for further details. Indeed, we distinguish two cases, on the one hand, if $y$ belongs to the interior of $(-\infty, 0], T_{(-\infty, 0]}(y)=\mathbb{R}$ and $F(t, y)=-2 p^{\prime}(t)$, we choose $c(t)=1+\left|2 p^{\prime}(t)\right|$ and on the other hand, if $y=0$, $T_{(-\infty, 0]}(0)=(-\infty, 0]$ and $F(t, y)=\left(-\infty,-2 p^{\prime}(t)\right]$, we choose $c(t)=1+\left|2 p^{\prime}(t)\right|$. Therefore the existence of solution to $(2.8)$ follows.

The uniqueness result comes from the monotonicity of $J_{N}$, namely $J_{N}$ is the subdifferential of a convex, lower semi-continuous and proper function, the reader is referred to [6] for further details.

Proof of Lemma 2.4. We note that

$$
\left(u^{\prime 2}+\dot{u}^{2}\right)^{\prime}-2 \frac{\partial}{\partial t}\left(u^{\prime} \dot{u}\right)=0 \text { in the sense of distributions. }
$$

Hence we integrate (A.1) over $\left[x_{0}, x_{1}\right] \times\left[t_{0}, t_{1}\right]$, with $0<x_{0}<x_{1}<L$ and $0<t_{0}<t_{1}<T$, to get

$$
\int_{t_{0}}^{t_{1}}\left(\left(u^{\prime 2}+\dot{u}^{2}\right)\left(x_{1}, t\right)-\left(u^{\prime 2}+\dot{u}^{2}\right)\left(x_{0}, t\right)\right) \mathrm{d} t=\int_{x_{0}}^{x_{1}}\left(\left(2 u^{\prime} \dot{u}\right)\left(x, t_{1}\right)-\left(2 u^{\prime} \dot{u}\right)\left(x, t_{0}\right)\right) \mathrm{d} x .
$$

According to Lemma 2.3, the right hand side of (A.2) is bounded independently of $x_{0}, x_{1}, t_{0}, t_{1}$ as long as $0<\bar{x}_{0} \leqslant x_{0}<x_{1}<L$. We integrate now (A.2) with respect to $x_{0}$ over $\left[\bar{x}_{0}, L\right]$, we may deduce that there exists $C>0$ independent of $x_{1}, \bar{x}_{0}$ such that

$$
\left(L-\bar{x}_{0}\right) \int_{t_{0}}^{t_{1}}\left(u^{\prime 2}+\dot{u}^{2}\right)\left(x_{1}, t\right) \mathrm{d} t \leq \int_{\bar{x}_{0}}^{L} \int_{t_{0}}^{t_{1}}\left(u^{\prime 2}+\dot{u}^{2}\right)\left(x_{0}, t\right) \mathrm{d} t \mathrm{~d} x_{0}+C\left(L-\bar{x}_{0}\right)
$$

which implies that $x \mapsto \int_{t_{0}}^{t_{1}}\left(u^{\prime 2}+\dot{u}^{2}\right)\left(x_{1}, t\right) \mathrm{d} t$ is bounded on $\left[\bar{x}_{0}, L\right]$ independently of $t_{0}$ and $t_{1}$, it follows that $x \mapsto \int_{0}^{T}\left(u^{\prime 2}+\dot{u}^{2}\right)(x, t) \mathrm{d} t$ is bounded on $\left[\bar{x}_{0}, L\right]$. Let $v$ be the solution of the following problem

$$
\left\{\begin{array}{l}
\ddot{v}-v^{\prime \prime}=0 \quad \text { on } \quad\left(0, x_{0}\right) \times(0, T), \\
v\left(x_{0}, t\right)=u\left(x_{0}, t\right) \quad \text { and } v^{\prime}\left(x_{0}, t\right)=u^{\prime}\left(x_{0}, t\right) \quad \text { for } \quad \text { all } \quad t \in[0, T], \\
v(x, 0)=u^{0}(x) \text { and } v(x, T)=u(x, T) \text { for all } \quad x \in\left[0, x_{0}\right] .
\end{array}\right.
$$

These conditions are illustrated in Figure 8. Since $u^{\prime}\left(x_{0}, \cdot\right)$ and $\dot{u}\left(x_{0}, \cdot\right)$ belong to $\mathrm{L}^{2}(0, T)$, we may infer that there exists a unique solution to (A.3). More precisely, $w=u-v$ satisfies (A.3) with homogeneous boundary initial conditions and the existence and uniqueness theorem in [4] holds. Furthermore $v=u$ on $\left(0, x_{0}\right) \times(0, T)$ and in particular we have $\dot{v}(x, 0)=v^{0}(x)$. We solve (A.3) by employing a classical characteristic method. To this aim, it is convenient to introduce the following notations:

$$
\beta_{1} \stackrel{\text { def }}{=}-x+t \quad \text { and } \quad \beta_{2} \stackrel{\text { def }}{=}-x-t .
$$

We may deduce that $\frac{\partial^{2} v}{\partial \beta_{1} \partial \beta_{2}}$ vanishes which implies that $v(x, t)=f\left(\beta_{1}\right)+g\left(\beta_{2}\right)$. Notice that the general solution for all of points in the rectangle $\left(0, x_{0}\right) \times(0, T)$ does not exist. Then we split the rectangle into three regions by 


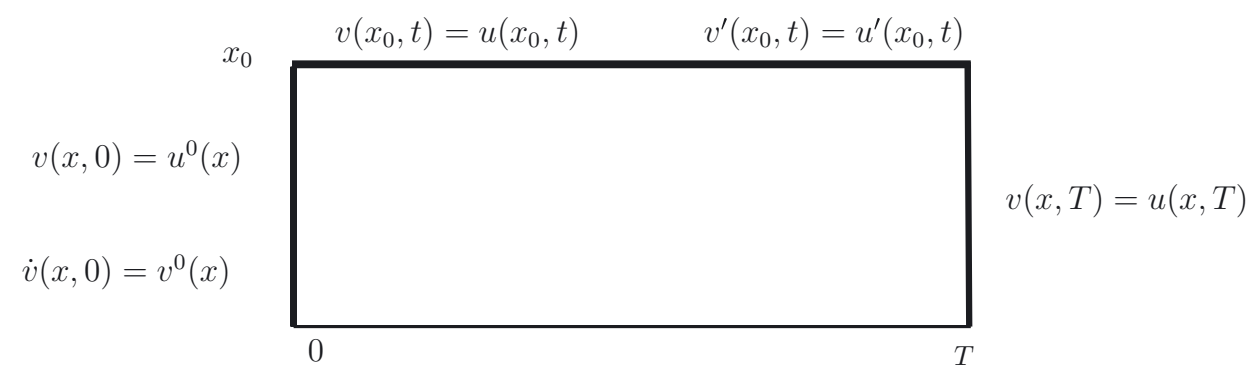

FiguRE 8. Initial and boundary conditions for $v$ on the rectangle $\left(0, x_{0}\right) \times(0, T)$.

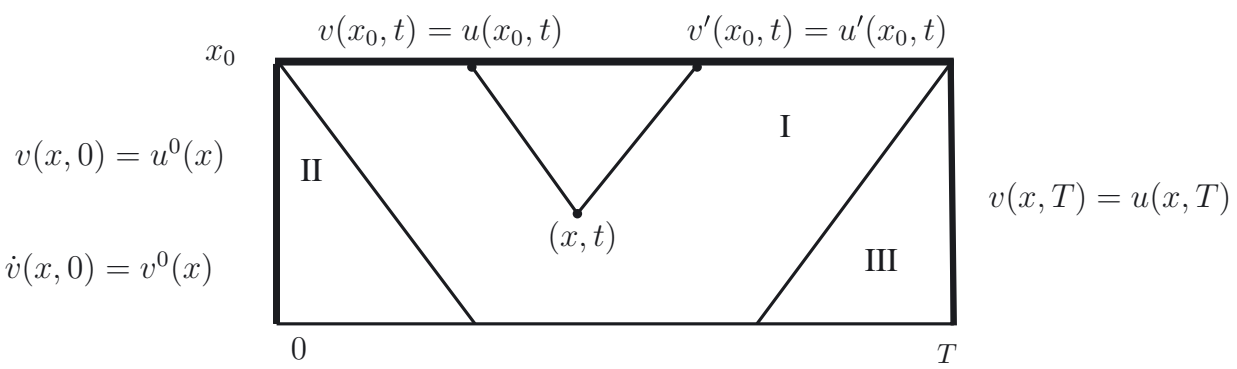

Figure 9. On characteristics in the region I.

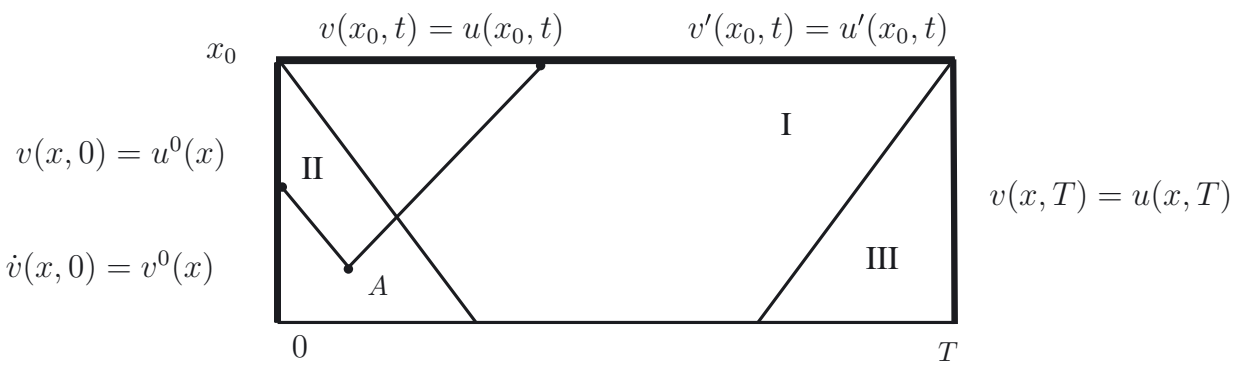

FiguRE 10. Characteristics in the region II.

using characteristic lines as it is shown on Figure 9. We looking for the solution in each region. More precisely, in region I and according to the initial condition of problem (A.3) in $x=x_{0}$ for $x \leq x_{0}$, we get

$$
v(x, t)=\frac{1}{2}\left(u\left(x_{0}, t+\left(x_{0}-x\right)\right)+u\left(x_{0}, t-\left(x_{0}-x\right)\right)\right)-\frac{1}{2} \int_{t-\left(x_{0}-x\right)}^{t+\left(x_{0}-x\right)} u^{\prime}\left(x_{0}, \zeta\right) \mathrm{d} \zeta .
$$

Observe that Figure 9 gives a better interpretation of this phenomenon. Indeed, the interval used will be the intersection of the line $x=x_{0}$ with the forward wave cone at $(x, t)$ which is the region between the two straight lines having a slope of \pm 1 but directed upwards from an origin $(x, t)$. The forward wave cone at the point $(x, t)$ will enclose all those points $\left(x_{0}, \zeta\right)$ which motion will be influenced by what occurred at the point $x$ at the time $t$.

Concerning the region II, we use the characteristics illustrated by Figure 10 . 
Let $A=\left(x_{A}, t_{A}\right)$ is a point in the region II. It follows that

$$
\begin{aligned}
& v^{\prime}\left(x_{A}, t_{A}\right)-\dot{v}\left(x_{A}, t_{A}\right)=u_{0}^{\prime}\left(x_{A}+t_{A}\right)-v^{0}\left(x_{A}+t_{A}\right), \\
& v^{\prime}\left(x_{A}, t_{A}\right)+\dot{v}\left(x_{A}, t_{A}\right)=u^{\prime}\left(x_{0}, t_{A}+\left(x_{0}-x_{A}\right)\right)+\dot{u}\left(x_{0}, t_{A}+\left(x_{0}-x_{A}\right)\right) .
\end{aligned}
$$

Therefore (A.5) leads to

$$
v^{\prime}\left(x_{A}, t_{A}\right)=\frac{1}{2}\left(u^{0^{\prime}}\left(x_{A}+t_{A}\right)-v^{0}\left(x_{A}+t_{A}\right)+u^{\prime}\left(x_{0}, t_{A}+\left(x_{0}-x_{A}\right)\right)+\dot{u}\left(x_{0}, t_{A}+\left(x_{0}-x_{A}\right)\right)\right),
$$

and

$$
\dot{v}\left(x_{A}, t_{A}\right)=\frac{1}{2}\left(-u^{0^{\prime}}\left(x_{A}+t_{A}\right)+v^{0}\left(x_{A}+t_{A}\right)+u^{\prime}\left(x_{0}, t_{A}+\left(x_{0}-x_{A}\right)\right)+\dot{u}\left(x_{0}, t_{A}+\left(x_{0}-x_{A}\right)\right)\right) .
$$

We have the solution for all the points located in the regions I and II. Concerning the solution in region III, we need some further regularity result to conclude. We obtained some regularity results for $u\left(x_{0}, t\right), u^{\prime}\left(x_{0}, t\right)$ and $\dot{u}\left(x_{0}, t\right)$ in Lemma 2.3. Besides by using (A.6) and (A.4), it is possible to deduce that $v^{\prime}$ belongs to $\mathrm{C}^{0}\left(\left[0, x_{0}\right] ; \mathrm{L}^{2}\left(0, T-x_{0}\right)\right)$ which implies that $v$ belongs to $\mathrm{C}^{0}\left(\left[0, x_{0}\right] ; \mathrm{H}^{1}\left(0, T-x_{0}\right)\right)$. As $x_{0}$ is arbitrary small, the conclusion is clear.

\section{Appendix B}

We establish below a Green's formula that is crucial in the proof of Lemma 3.2. To this aim, let us introduce a linear topological space $\mathcal{D}$ and Hilbert spaces $\mathcal{V}, \mathcal{H}, \mathcal{Z}$ and $\mathcal{S}$ with their topological duals denoted by $\mathcal{D}^{\prime}, \mathcal{V}^{\prime}, \mathcal{H}^{\prime}, \mathcal{Z}^{\prime}$ and $\mathcal{S}^{\prime}$.

Suppose that $\mathcal{D}$ is contained in $\mathcal{V}$ and it is dense in $\mathcal{H}$. Here $\mathcal{H}$ is identified with its dual, namely we have $\mathcal{H}=\mathcal{H}^{\prime}$. Furthermore, Suppose that $\mathcal{V}$ is contained in $\mathcal{H}$ with finer topology and we denote by $\mathcal{V}_{0}$, the closure of $\mathcal{D}$ in $\mathcal{V}$ such that

$$
\mathcal{D} \hookrightarrow \mathcal{V}_{0} \hookrightarrow \mathcal{H}=\mathcal{H}^{\prime} \hookrightarrow \mathcal{V}_{0}^{\prime}
$$

with dense embedding. More precisely, $\mathcal{D}$ is an abstraction of the usual space $\mathcal{D}\left(Q_{T}\right)$ of test function. In our case the spaces $\mathcal{V}, \mathcal{S}$ and $\mathcal{Z}$ denote the admissible displacement, stresses and boundary values containing traces of element of $\mathcal{V}$, respectively. We also introduce a linear operator $A \in \mathcal{L}(\mathcal{V}, \mathcal{S})$ and its restriction to $\mathcal{V}_{0}$ denoted by $A_{0} \in \mathcal{L}\left(\mathcal{V}_{0}, \mathcal{S}\right)$ such that

$$
\forall v \in \mathcal{V}_{0}: A v=A_{0} v
$$

Let $A^{*} \in \mathcal{L}\left(\mathcal{S}^{\prime}, \mathcal{V}^{\prime}\right)$ be the adjoint of the operator $A$, defined by

$$
\langle\tau, A v\rangle_{\mathcal{S}^{\prime} \times \mathcal{S}}=\left\langle A^{*} \tau, v\right\rangle_{\mathcal{V}^{\prime} \times \mathcal{V}}
$$

Then

$$
\forall \tau \in \mathcal{S}^{\prime}, \forall v \in \mathcal{V}_{0}: \quad\langle\tau, A v\rangle_{\mathcal{S}^{\prime} \times \mathcal{S}}=\left\langle A_{0}^{*} \tau, v\right\rangle_{\mathcal{V}^{\prime} \times \mathcal{V}}
$$

Let us define $\mathcal{S}^{\prime}\left(A_{0}^{*}\right) \stackrel{\text { def }}{=}\left\{\tau \in \mathcal{S}^{\prime}: A_{0}^{*} \tau \in H\right\}$, then we have the following trace theorem.

Theorem 5.1. Suppose that $\mathcal{V}_{0}$ is the kernel of a surjective map $\gamma \in \mathcal{L}(\mathcal{V}, \mathcal{Z})$ from $\mathcal{V}$ onto $\mathcal{Z}$. Then there exists a unique linear operator $\pi \in \mathcal{L}\left(\mathcal{S}^{\prime}\left(A_{0}^{*}\right), \mathcal{Z}^{\prime}\right)$ such that the following Green's formula holds:

$$
\forall \tau \in \mathcal{S}^{\prime}\left(A_{0}^{*}\right), \forall v \in \mathcal{V}: \quad\langle\tau, A v\rangle_{\mathcal{S}^{\prime} \times \mathcal{S}}-\left\langle A_{0}^{*} \tau, v\right\rangle_{\mathcal{V}^{\prime} \times \mathcal{V}}=\langle\pi \tau, \gamma v\rangle_{\mathcal{Z}^{\prime} \times \mathcal{Z}} .
$$

The detailed proof of (5.1) is given in [3]. In particular, we are interested in this work to the case where $\mathcal{S}=$ $\mathcal{S}^{\prime} \stackrel{\text { def }}{=}\left\{\left(u_{1}, u_{2}\right) \in \mathrm{L}^{2}\left(Q_{T}\right)\right\}, \mathcal{D} \stackrel{\text { def }}{=} \mathcal{D}\left(Q_{T}\right), \mathcal{V} \stackrel{\text { def }}{=} \mathrm{H}^{1}\left(Q_{T}\right), \mathcal{V}_{0}=\mathrm{H}_{0}^{1}\left(Q_{T}\right), \mathcal{V}_{0}^{\prime}=\mathrm{H}^{-1}\left(Q_{T}\right), \mathcal{H} \stackrel{\text { def }}{=} \mathrm{L}^{2}\left(Q_{T}\right), \mathcal{S}^{\prime}\left(A_{0}^{*}\right)=$ $\left\{\left(u_{1}, u_{2}\right) \in \mathcal{S}: \frac{\partial}{\partial t} u_{1}-\frac{\partial}{\partial x} u_{2} \in \mathrm{L}^{2}\left(Q_{T}\right)\right.$ in the sense of distributions $\}, A: u \mapsto\left(\frac{\partial}{\partial t} u,-\frac{\partial}{\partial x} u\right), \mathcal{Z} \stackrel{\text { def }}{=} \mathrm{H}^{1 / 2}\left(\partial Q_{T}\right)$ and 
$\mathcal{Z}^{\prime}=\mathrm{H}^{-1 / 2}\left(\partial Q_{T}\right)$, where $\left(\partial Q_{T}\right)$ is the boundary of $Q_{T}$ and the trace operator $\gamma: \mathcal{V} \rightarrow \mathcal{Z}$. Then there exists a unique $\pi \in \mathcal{L}\left(\mathcal{S}^{\prime}\left(A_{0}^{*}\right), \mathcal{Z}^{\prime}\right)$ such that

$$
\int_{Q_{T}}\left(\tau_{1} \frac{\partial}{\partial t} v+\tau_{2} \frac{\partial}{\partial x} v\right) \mathrm{d} t \mathrm{~d} x-\int_{Q_{T}}\left(\frac{\partial}{\partial t} \tau_{1}-\frac{\partial}{\partial x} \tau_{2}\right) v \mathrm{~d} t \mathrm{~d} x=\langle\pi \tau, \gamma v\rangle_{\mathcal{Z}^{\prime} \times \mathcal{Z}},
$$

for all $v \in \mathcal{V}$ and $\left(\tau_{1}, \tau_{2}\right) \in \mathcal{S}^{\prime}\left(A_{0}^{*}\right)$. Then by density argument, we have

$$
\langle\pi \tau, \gamma v\rangle_{\mathcal{Z}^{\prime} \times \mathcal{Z}}=\int_{\partial Q_{T}}(\tau . n) v \mathrm{~d} s
$$

where $n$ is the outward unit normal to $Q_{T}$, when $\tau$ and $v$ are regular enough.

\section{REFERENCES}

[1] P. Alart and A. Curnier, A generalized Newton method for contact problems with friction. J. Mech. Theor. Appl. 7 (1988) 67-82.

[2] F. Armero and E. Petocz, Formulation and analysis of conserving algorithms for frictionless dynamic contact/impact problems. Comput. Methods Appl. Mech. Engrg. 158 (1998) 269-300.

[3] J.P. Aubin, Approximation of elliptic boundary-value problems. Pure and Applied Mathematics, Vol. XXVI. Wiley-Interscience (1972).

[4] J.M. Ball, Strongly continuous semigroups, weak solutions, and the variation of constants formula. Proc. Amer. Math. Soc. 63 (1977) 370-373.

[5] D. Bárcenas, The fundamental theorem of calculus for Lebesgue integral. Divulg. Mat. 8 (2000) 75-85.

[6] H. Brezis, Opérateurs maximaux monotones et semi-groupes de contractions dans les espaces de Hilbert. North-Holland Mathematics Studies, No. 5. Notas de Matemática (50). North-Holland Publishing Co., Amsterdam (1973).

[7] M. Crouzeix and A.L. Mignot, Analyse numérique des équations différentielles. Collection Mathématiques Appliquées pour la Maîtrise. Masson, Paris (1984).

[8] R. Dautray and J.-L. Lions, Analyse mathématique et calcul numérique pour les sciences et les techniques. Vol. 8. INSTN: Collection Enseignement. Masson, Paris (1988).

[9] K. Deimling, Multivalued differential equations. Vol. 1 of de Gruyter Series in Nonlinear Analysis and Applications. Walter de Gruyter \& Co., Berlin (1992).

[10] D. Doyen and A. Ern, Convergence of a space semi-discrete modified mass method for the dynamic Signorini problem. Commun. Math. Sci. 7 (2009) 1063-1072.

[11] D. Doyen, A. Ern and S. Piperno, Time-integration schemes for the finite element dynamic Signorini problem. SIAM J. Sci. Comput. (2011) 223-249.

[12] A. Ern and J.L. Guermond, Theory and practice of finite elements. Appl. Math. Sci., vol. 159. Springer-Verlag, New York (2004).

[13] C. Hager and B.I. Wohlmuth, Analysis of a space-time discretization for dynamic elasticity problems based on mass-free surface elements. SIAM J. Num. Anal. 47 (2009) 1863-1885.

[14] P. Hauret, Mixed interpretation and extensions of the equivalent mass matrix approach for elastodynamics with contact. Comput. Methods Appl. Mech. Engrg. 199 (2010) 2941-2957.

[15] T.J.R. Hugues, R.L. Taylor, J.L. Sackman, A. Curnier and W. Kano Knukulchai, A finite method for a class of contact-impact problems. Comput. Methods Appl. Mech. Engrg. 8 (1976) 249-276.

[16] H.B. Khenous, P. Laborde and Y. Renard, Mass redistribution method for finite element contact problems in elastodynamics. Eur. J. Mech. A Solids 27 (2008) 918-932.

[17] S. Krenk, Energy conservation in Newmark based time integration algorithms. Comput. Methods Appl. Mech. Engrg. 195 (2006) 6110-6124.

[18] N. Kikuchi and J.T. Oden, Contact problems in elasticity: a study of variational inequalities and finite element methods. SIAM Studies Appl. Math. SIAM, Philadelphia, Pa (1988).

[19] J.U. Kim A boundary thin obstacle problem for a wave equation. Commun. Partial Differential Eqs. 14 (1989) $1011-1026$.

[20] T.A. Laursen and V. Chawla, Design of energy conserving algorithms for frictionless dynamic contact problems. Int. J. Numer. Methods Engrg. 40 (1997) 863-886.

[21] T.A. Laursen and G.R. Love, Improved implicit integrators for transient impact problems-geometric admissibility within the conserving framework. Int. J. Numer. Methods Engrg. 53 (2002) 245-274.

[22] G. Lebeau and M. Schatzman, A wave problem in a half-space with a unilateral constraint at the boundary. J. Differ. Eqs. 53 (1984) 309-361.

[23] J.-J. Moreau, Liaisons unilatérales sans frottement et chocs inélastiques. C. R. Acad. Sci. Paris Sér. II Méc. Phys. Chim. Sci. Univers Sci. Terre 296 (1983) 1473-1476. 
[24] J.-J. Moreau and P.D. Panagiotopoulos, Nonsmooth mechanics and applications. Vol. 302 of CISM Courses Lect. SpringerVerlag, Vienna (1988).

[25] L. Paoli, Time discretization of vibro-impact. R. Soc. London Philos. Trans. Ser. A Math. Phys. Eng. Sci. 359 (2001) 24052428.

[26] L. Paoli and M. Schatzman, A numerical scheme for impact problem I. SIAM J. Numer. Anal. 40 (2002) $702-733$.

[27] L. Paoli and M. Schatzman, Approximation et existence en vibro-impact. C. R. Acad. Sci. Paris Sér. I Math. 329 (1999) 1003-1007.

[28] Y. Renard, Generalized Newton's methods for the approximation and resolution of frictional contact problems in elasticity. Comput. Meth. Appl. Mech. Engng. 256 (2013) 38-55.

[29] Y. Renard and J. Pommier, Getfem++. An Open Source generic C++ library for finite element methods. http://home.gna.org/getfem.

[30] W. Rudin, Real and complex analysis. McGraw-Hill Series in Higher Mathematics. McGraw-Hill Book Co., New York, 2nd edn (1974).

[31] M. Schatzman, A hyperbolic problem of second order with unilateral constraints: the vibrating string with a concave obstacle. J. Math. Anal. Appl. 73 (1980) 138-191.

[32] M. Schatzman and M. Bercovier, Numerical approximation of a wave equation with unilateral constraints. Math. Comput. $\mathbf{5 3}$ (1989) 55-79.

[33] K. Schweizerhof, J.O. Hallquist and D. Stillman, Efficiency refinements of contact strategies and algorithms in explicit finite element programming. Compt. Plasticity. Edited by Owen, Onate, Hinton, Pineridge (1992) 457-482.

[34] J. Simon, Compact sets in the space L ${ }^{p}(0, T ; B)$, Ann. Mat. Pura Appl. 146 (1987) 65-96.

[35] P. Wriggers, Computational contact mechanics. John Wiley and Sons Ltd. (2002). 